\title{
An $E_{8}$-approach to the moonshine vertex operator algebra
}

\author{
Hiroki SHIMAKURA* \\ Department of Mathematics, \\ Aichi University of Education, \\ 1 Hirosawa, Igaya-cho, Kariya-city, Aichi, 448-8542 Japan \\ e-mail: shima@auecc.aichi-edu.ac.jp \\ 2000 Mathematics Subject Classification. Primary 17B69; Secondary 20 D08.
}

\begin{abstract}
In this article, we study the moonshine vertex operator algebra starting with the tensor product of three copies of the vertex operator algebra $V_{\sqrt{2} E_{8}}^{+}$, and describe it by the quadratic space over $\mathbb{F}_{2}$ associated to $V_{\sqrt{2} E_{8}}^{+}$. Using quadratic spaces and orthogonal groups, we show the transitivity of the automorphism group of the moonshine vertex operator algebra on the set of all full vertex operator subalgebras isomorphic to the tensor product of three copies of $V_{\sqrt{2} E_{8}}^{+}$, and determine the stabilizer of such a vertex operator subalgebra. Our approach is a vertex operator algebra analogue of "An $E_{8}$-approach to the Leech lattice and the Conway group" by Lepowsky and Meurman. Moreover, we find new analogies among the moonshine vertex operator algebra, the Leech lattice and the extended binary Golay code.
\end{abstract}

\section{Introduction}

The moonshine vertex operator algebra (VOA) is one of the most important VOAs. A reason is that its automorphism group is isomorphic to the Monster, the largest sporadic finite simple group. Hence the Monster can be studied as symmetries of the moonshine VOA.

The moonshine VOA was constructed by Frenkel-Lepowsky-Meurman [FLM88] from the Leech lattice as a VOA analogue of the construction of the Leech lattice from the extended binary Golay code. Hence, it is natural to regard VOAs as analogues of binary codes and lattices. In fact, by this approach, the upper bound of the minimum conformal weight of a holomorphic VOA was given in [Hö95], and the notion of a conformal design based on a VOA was introduced in [Hö08]. For other example, some isomorphism problems of VOAs were solved in $[\mathrm{Sh}]$.

Lepowsky and Meurman studied the Leech lattice in [LM82] starting with the orthogonal direct sum of three copies of the $E_{8}$ root lattice, and described it by the quadratic space over $\mathbb{F}_{2}$ associated to the $E_{8}$ root lattice. This is a lattice analogue of Turyn's construction of the extended binary Golay code (cf. [MS77]). These are useful for understanding the automorphism

*The author was partially supported by Grants-in-Aid for Scientific Research (No. 20549004) and Excellent Young Researcher Overseas Visit Program, Japan Society for the Promotion of Science. 
groups of the Golay code and the Leech lattice, the Mathieu group and the Conway group ([CS99, LM82, Gr99]). Hence it is really important for the study of the Monster to find a VOA analogue of these descriptions.

In this article, we study the moonshine VOA starting with the tensor product of three copies of the VOA $V_{\sqrt{2} E_{8}}^{+}$, and describe it as a simple current extension of $\left(V_{\sqrt{2} E_{8}}^{+}\right)^{\otimes 3}$ by using a certain maximal totally singular subspace of the quadratic space over $\mathbb{F}_{2}$ associated to $\left(V_{\sqrt{2} E_{8}}^{+}\right)^{\otimes 3}$. This is a VOA analogue of Lepowsky and Meurman's description of the Leech lattice. Note that the moonshine VOA was already constructed by Miyamoto [Mi04 as a simple current extension of $\left(V_{\sqrt{2} E_{8}}^{+}\right)^{\otimes 3}$ without quadratic spaces.

An advantage of our description is that we can effectively apply results of quadratic spaces and orthogonal groups to VOAs. The transitivity of the automorphism group of the moonshine VOA on the set of all full subVOAs isomorphic to $\left(V_{\sqrt{2} E_{8}}^{+}\right)^{\otimes 3}$ is proved by the uniqueness of certain maximal totally singular subspaces of the quadratic space, and a subgroup of the automorphism group of the moonshine VOA of shape $2^{15} \cdot\left(2^{20}:\left(\mathrm{SL}_{5}(2) \times \mathrm{Sym}_{3}\right)\right)$ is described as a lift of a certain subgroup of the orthogonal group. According to [CCNPW85, a subgroup with this shape is a maximal 2-local in the Monster.

Another advantage is that we can find some aspects of the moonshine VOA and the Monster analogous to the Leech lattice and the Conway group, and to the extended binary Golay code and the Mathieu group respectively. For example, the transitivity in the previous paragraph is a VOA analogue of that of the Mathieu group on the set of all trios of the extended binary Golay code (cf. [CS99]) and that of the Conway group on the set of all sublattices of the Leech lattice isomorphic to $\left(\sqrt{2} E_{8}\right)^{\oplus 3}$ ([Gr99]). Moreover, the similarity of the following shapes of the stabilizers of a trio in the Mathieu group, of $\left(\sqrt{2} E_{8}\right)^{\oplus 3}$ in the Conway group, and of $\left(V_{\sqrt{2} E_{8}}^{+}\right)^{\otimes 3}$ in the Monster is explained by the associated orthogonal groups and quadratic spaces:

$$
2^{6}:\left(\mathrm{SL}_{3}(2) \times \operatorname{Sym}_{3}\right), 2^{3} \cdot\left(2^{12}:\left(\mathrm{SL}_{4}(2) \times \operatorname{Sym}_{3}\right)\right), 2^{15} \cdot\left(2^{20}:\left(\mathrm{SL}_{5}(2) \times \operatorname{Sym}_{3}\right)\right) .
$$

As another example, we find the following equation for the dimension of the weight 2 subspace of the moonshine VOA (Proposition 4.15):

$$
(3 \times 156)+\left(3 \times\left(2^{5}-1\right)\right) \times 1^{0} \times 8^{2}+\left(3 \times\left(2^{5}-1\right) \times 2^{8}\right) \times 1^{2} \times 8^{1}=196884,
$$

where 156 is the dimension of the weight 2 subspace of the $\operatorname{VOA} V_{\sqrt{2} E_{8}}^{+}$. This is a VOA analogue of the following equations for the number of octads in the extended binary Golay code and that of vectors of norm 4 in the Leech lattice:

$$
\begin{array}{ccccc}
(3 \times 1) & +\left(3 \times\left(2^{3}-1\right)\right) \times 1^{0} \times 2^{2}+\left(3 \times\left(2^{3}-1\right) \times 2^{4}\right) \times 1^{2} \times 2^{1}= & 759, \\
(3 \times 240)+\left(3 \times\left(2^{4}-1\right)\right) \times 2^{0} \times 16^{2} & +\left(3 \times\left(2^{4}-1\right) \times 2^{6}\right) \times 2^{2} \times 16^{1}= & 196560 .
\end{array}
$$

Let us explain the main results. First, we discuss quadratic spaces over $\mathbb{F}_{2}$, which is crucial for this article. Let $(R, q)$ be a non-singular quadratic space of plus type over $\mathbb{F}_{2}$ and let $w: R \rightarrow\{0,1,2\}$ be the map defined by setting

$$
w(a)= \begin{cases}0 & \text { if } v=0, \\ 1 & \text { if } q(a)=1, \\ 2 & \text { if } q(a)=0 \text { and } v \neq 0 .\end{cases}
$$

Let $\left(R^{3}, q^{3}\right)$ be the orthogonal direct sum of three copies of $(R, q)$ and let $\mathcal{S}$ be a maximal totally singular subspace of $\left(R^{3}, q^{3}\right)$ satisfying

$$
w^{3}(v) \geq 4, \quad \forall v \in \mathcal{S} \backslash\{0\},
$$


where $w^{3}\left(a_{1}, a_{2}, a_{2}\right)=\sum_{i=1}^{3} w\left(a_{i}\right)$. Such a subspace was constructed in [LM82] from complementary maximal totally singular subspaces of $(R, q)$. We show in Theorem 2.8 that $\mathcal{S}$ is unique up to $\operatorname{Aut}\left(R^{3}, w^{3}\right)$, the subgroup of the orthogonal group $O\left(R^{3}, q^{3}\right)$ preserving $w^{3}$, and determine in Proposition 2.5 the stabilizer of $\mathcal{S}$ in $\operatorname{Aut}\left(R^{3}, w^{3}\right)$.

Next, we describe the moonshine VOA by the quadratic space. Let $V=V_{\sqrt{2} E_{8}}^{+}$and let $R(V)$ denote the set of all isomorphism classes of irreducible $V$-modules. By [AD04, ADL05, $R(V)$ has a 10-dimensional vector space structure over $\mathbb{F}_{2}$ under the fusion rules. By [Sh04, $R(V)$ has a non-singular quadratic form $q_{V}$ of plus type, and hence $\left(R(V), q_{V}\right)$ is a 10-dimensional nonsingular quadratic space of plus type over $\mathbb{F}_{2}$. Let $\mathcal{S}$ be a maximal totally singular subspace of $\left(R(V)^{3}, q_{V}^{3}\right)$. We identify $R(V)^{3}$ with $R\left(V^{3}\right)$ ([FHL93, DMZ94]), where $V^{3}$ is the tensor product of three copies of $V$. Then by a theory of simple current extensions ([Hu96, Mi04, LY08]), the $V^{3}$-module $\mathfrak{V}(\mathcal{S})=\oplus_{[M] \in \mathcal{S}} M$ has a holomorphic VOA structure, where $[M]$ is the isomorphism class of $M$. In this case, for $[M] \in R\left(V^{3}\right)$, the lowest weight of $M$ is $w^{3}([M]) / 2$. Assume that $\mathcal{S}$ satisfies the condition on $w^{3}$ in the previous paragraph. Then the the weight 1 subspace of $\mathfrak{V}(\mathcal{S})$ is trivial. Since $V$ is framed, so is $\mathfrak{V}(\mathcal{S})$. Hence by [LY07, $\mathfrak{V}(\mathcal{S})$ is isomorphic to the moonshine VOA (Theorem 4.10).

Finally, we study the moonshine VOA and the automorphism group by using the orthogonal group and the quadratic space. By [Sh04], the stabilizer of $\mathcal{S}$ in $\operatorname{Aut}\left(V^{3}\right)\left(\cong \operatorname{Aut}\left(R(V)^{3}, w^{3}\right)\right)$ lifts to a subgroup of $\operatorname{Aut}(\mathfrak{V}(\mathcal{S}))$, and its shape is $2^{15} \cdot\left(2^{20}:\left(\mathrm{SL}_{5}(2) \times \mathrm{Sym}_{3}\right)\right)$. The uniqueness of $\mathcal{S}$ up to $\operatorname{Aut}\left(R(V)^{3}, w^{3}\right)$ implies the transitivity of $\operatorname{Aut}(\mathfrak{V}(\mathcal{S}))$ on the set of all full subVOAs of $\mathfrak{V}(\mathcal{S})$ isomorphic to $\left(V_{\sqrt{2} E_{8}}^{+}\right)^{3}$ (Theorem 4.17). Moreover, we describe in Proposition 4.15 the dimension of the weight 2 subspace in terms of quadratic spaces.

In order to compare VOAs to binary codes and lattices, we study the extended binary Golay code and the Leech lattice by similar approaches in Section 3, which were already discussed in [MS77, CS99, LM82, Gr99, Gr].

\section{Notations}

$\begin{array}{ll}\langle,\rangle & \text { The symplectic form on } R \text { or on } R^{k} . \\ (,) & \text { A positive definite bilinear form on } \mathbb{R}^{n} \text { or the standard inner product on } \mathbb{F}_{2}^{n} . \\ \times & \text { The fusion rules for a VOA. } \\ 2^{n} & \text { An elementary abelian 2-group of order } 2^{n} . \\ A . B & \text { A group extension with normal subgroup } A \text { and quotient } B . \\ A: B & \text { A split extension with normal subgroup } A \text { and quotient } B . \\ C^{k} & \text { The direct sum of } k \text { copies of a binary code } C . \\ \mathfrak{C}(\mathcal{S}) & \text { The binary code associated to } \mathcal{S} \subset R(C)^{k} . \\ E_{8} & \text { The } E_{8} \text { root lattice. } \\ \Phi, \Psi & \text { Maximal totally singular subspaces of } R, R(C), R(L) \text { or } R(V) . \\ \Phi_{(i j)} & \Phi_{(i j)}=\left\{\left(a_{1}, a_{2}, \ldots, a_{k}\right) \in R^{k} \mid a_{i}=a_{j} \in \Phi, a_{l}=0 \text { if } l \neq i, j\right\} . \\ \Psi_{(12 \ldots k)} & \Psi_{(12 \ldots k)}=\left\{(b, \ldots, b) \in R^{k} \mid b \in \Psi\right\} \\ (\Phi \cap \Psi)_{(1)} & (\Phi \cap \Psi)_{(1)}=\left\{(a, 0, \ldots, 0) \in R^{k} \mid a \in \Phi \cap \Psi\right\} . \\ g \circ M & \text { The conjugate of a module } M \text { for a } \operatorname{VOA} \text { by an automorphism } g . \\ g \circ[M] & \text { The isomorphism class of } g \circ M . \\ L^{k} & \text { The orthogonal direct sum of } k \text { copies of a lattice } L . \\ \mathfrak{L}(\mathcal{S}) & \text { The lattice associated to } \mathcal{S} \subset R(L)^{k} . \\ {[M]} & \text { The isomorphism class of a module } M \text { for a VOA. } \\ O(R, q) & \text { The orthogonal group of a quadratic space }(R, q) . \\ O_{2}(G) & \text { The maximal normal } 2 \text {-subgroup of a group } G . \\ \rho_{i} & \text { The } i \text {-th coordinate projection from } R^{k} \text { to } R . \\ q & \text { A quadratic form on } R \text { of plus type } \\ q C & \text { The quadratic form on } R(C) \text { defined by } q C(x)=\text { wt }(x) / 2(\bmod 2) .\end{array}$




$\begin{array}{ll}q_{L} & \text { The quadratic form on } R(L) \text { defined by } q_{L}(x)=(x, x)(\bmod 2) . \\ q_{V} & \text { The quadratic form on } R(V) \text { defined by } \\ & q_{V}([M])=0,1 \text { if } M \text { is } \mathbb{Z} \text {-graded, }(\mathbb{Z}+1 / 2) \text {-graded respectively. } \\ \mathrm{SL}_{n}(2) & \text { The special linear group of degree } n \text { over } \mathbb{F}_{2} . \\ \mathrm{Sym}_{n} & \text { The symmetric group of degree } n . \\ \mathcal{S} & \text { A maximal totally singular subspace of } R^{k} . \\ \mathcal{S}(\Phi, \Psi ; k) & \text { The maximal totally singular subspace of } R^{k} \\ & \text { spanned by } \Phi_{(1 i)}(2 \leq i \leq k), \Psi_{(12 \ldots)} \text { and }(\Phi \cap \Psi)_{(1)} . \\ (R, q) & \text { A non-singular quadratic space of plus type with } q \text { over } \mathbb{F}_{2} . \\ \left(R^{k}, q^{k}\right) & \text { The orthogonal direct sum of } k \text { copies of }(R, q) . \\ R(C) & C^{\perp} / C, \text { where } C^{\perp} \text { is the dual code of } C . \\ R(L) & L^{*} / L, \text { where } L^{*} \text { is the dual lattice of } L . \\ R(V) & \text { The set of all isomorphism classes of irreducible modules for a VOA } V . \\ V & \text { A simple rational } C_{2} \text {-cofinite self-dual VOA of CFT type, or } V=V_{\sqrt{2}}^{+} E_{8} . \\ V^{k} & \text { The tensor product of } k \text { copies of a VOA } V . \\ \mathfrak{V}(\mathcal{S}) & \text { The VOA associated to } \mathcal{S} \subset R(V)^{k} . \\ w^{k} & \text { The map from } R^{k} \text { to }\{0,1, \ldots, 2 k\}(\text { see Section } 2.1) . \\ \mathrm{wt}(x) & \text { The (Hamming) weight of } x=\left(x_{i}\right) \in \mathbb{F}_{2}^{n} \text { defined by wt }(x)=\left|\left\{i \mid x_{i} \neq 0\right\}\right| .\end{array}$

\section{Preliminary}

In this section, we recall or give some definitions and facts necessary in this article.

\subsection{Quadratic spaces and orthogonal groups}

Let us recall fundamental facts on quadratic spaces over $\mathbb{F}_{2}$ and orthogonal groups (cf. [Ch97]).

Let $R$ be a $2 m$-dimensional vector space over $\mathbb{F}_{2}$. A form $\langle\rangle:, R \times R \rightarrow \mathbb{F}_{2}$ is said to be symplectic if it is a symmetric bilinear form with $\langle a, a\rangle=0$ for all $a \in R$. A map $q: R \rightarrow \mathbb{F}_{2}$ is called a quadratic form on $R$ if the associated form defined by $\langle a, b\rangle=q(a+b)+q(a)+q(b)$, $a, b \in R$, is symplectic. A quadratic form is said to be non-singular if the associated symplectic form is non-singular, that is, $R^{\perp}=\{a \in R \mid\langle a, R\rangle=0\}=0$. The pair $(R, q)$ consisting of a vector space $R$ over $\mathbb{F}_{2}$ and a quadratic form $q$ on it is called a quadratic space, and it is said to be non-singular if $q$ is non-singular. A vector $a \in R$ is said to be singular (resp. non-singular) if $q(a)=0$ (resp. $q(a)=1$ ). A subspace $\Phi$ of $R$ is said to be totally singular if any vector in $\Phi$ is singular. A non-singular quadratic form $q$ is said to be of plus type if the dimension of a maximal totally singular subspace of $(R, q)$ is $m$. Let $O(R, q)$ denote the orthogonal group of $(R, q)$, the subgroup of $\mathrm{GL}(R)$ preserving $q$. The following lemma is well-known.

Lemma 1.1. (cf. [Ch97]) Let $(R, q)$ be a non-singular 2m-dimensional quadratic space of plus type over $\mathbb{F}_{2}$. Let $\Phi$ be a maximal totally singular subspace of $R$ and let $H$ be the stabilizer of $\Phi$ in $O(R, q)$.

(1) The subspace of $R$ orthogonal to $\Phi$ is equal to $\Phi$, that is, $\Phi^{\perp}=\Phi$.

(2) The orthogonal group $O(R, q)$ is transitive on the set of all maximal totally singular subspaces of $R$.

(3) The maximal normal 2-subgroup $\mathrm{O}_{2}(H)$ of $H$ acts trivially on both $\Phi$ and $R / \Phi$, and $H / \mathrm{O}_{2}(H)$ acts on $\Phi$ as $\mathrm{SL}(\Phi)\left(\cong \mathrm{SL}_{m}(2)\right)$. Moreover, $H$ has the shape $2^{\left(\begin{array}{c}m \\ 2\end{array}\right)}: \mathrm{SL}_{m}(2)$.

(4) There exists a maximal totally singular subspace $\Psi$ of $R$ such that $\Phi \cap \Psi=0$. Moreover, the stabilizer of $\Psi$ in $H$ is isomorphic to $\mathrm{SL}_{m}(2)$. 
(5) All pairs of complementary maximal totally singular subspaces of $R$ are conjugate under $O(R, q)$.

(6) $O(R, q)$ is transitive on the set of all non-zero singular vectors in $R$.

\section{$1.2 \quad$ Vertex operator algebras}

In this subsection, we recall the definitions of vertex operator algebras (VOAs) and modules from [Bo86, FLM88, FHL93]. Throughout this article, all VOAs are defined over the field $\mathbb{C}$ of complex numbers unless otherwise stated.

A vertex operator algebra $(\mathrm{VOA})(V, Y, \mathbf{1}, \omega)$ is a $\mathbb{Z}_{\geq 0}$-graded vector space $V=\oplus_{m \in \mathbb{Z}_{\geq 0}} V_{m}$ equipped with a linear map

$$
Y(a, z)=\sum_{i \in \mathbb{Z}} a_{i} z^{-i-1} \in(\operatorname{End}(V))\left[\left[z, z^{-1}\right]\right], \quad a \in V,
$$

the vacuum vector 1 and the Virasoro element $\omega$ satisfying a number of conditions (Bo86, FLM88]). We often denote it by $V$ or $(V, Y)$ simply.

Two VOAs $(V, Y, \mathbf{1}, \omega)$ and $\left(V^{\prime}, Y^{\prime}, \mathbf{1}^{\prime}, \omega^{\prime}\right)$ are said to be isomorphic if there exists a linear isomorphism $g$ from $V$ to $V^{\prime}$ satisfying

$$
g \omega=\omega^{\prime} \quad \text { and } \quad g Y(v, z)=Y^{\prime}(g v, z) g \quad \text { for } \forall v \in V .
$$

When two VOAs are the same, such a linear isomorphism is called an automorphism. The group of all automorphisms of $V$ is called the automorphism group of $V$ and is denoted by $\operatorname{Aut}(V)$. We mean by a subVOA (or a vertex operator subalgebra) a graded subspace of $V$ which has a structure of a VOA such that the operations and its grading agree with the restriction of those of $V$ and that they share the vacuum vector. When they share also the Virasoro element, we will call it a full subVOA.

An (ordinary) module $\left(M, Y_{M}\right)$ for a VOA $V$ is a $\mathbb{C}$-graded vector space $M=\oplus_{m \in \mathbb{C}} M_{m}$ equipped with a linear map

$$
Y_{M}(a, z)=\sum_{i \in \mathbb{Z}} a_{i} z^{-i-1} \in(\operatorname{End}(M))\left[\left[z, z^{-1}\right]\right], \quad a \in V
$$

satisfying a number of conditions ([FHL93]). We often denote it by $M$ and its isomorphism class by $[M]$. The weight of a homogeneous vector $v \in M_{k}$ is $k$. If $M$ is irreducible then there exists the lowest weight $h \in \mathbb{C}$ of $M$ such that $M=\oplus_{m \in \mathbb{Z}_{\geq 0}} M_{h+m}$ and $M_{h} \neq 0$.

A VOA $V$ is said to be of CFT type if $V_{0}=\mathbb{C} 1$, is said to be rational if any module is completely reducible, and is said to be $C_{2}$-cofinite if $V / \operatorname{Span}_{\mathbb{C}}\left\{a_{-2} b \mid a, b \in V\right\}$ is finitedimensional. A VOA is said to be holomorphic if it is the only irreducible module up to isomorphism. A module $M$ is said to be self-dual if its contragredient module (cf. [FHL93]) is isomorphic to itself. Let $R(V)$ denote the set of all isomorphism classes of irreducible $V$ modules. Note that if $V$ is rational then $|R(V)|<\infty$.

Let $M_{a}, M_{b}, M_{c}$ be modules for a simple rational $C_{2}$-cofinite VOA $V$. An intertwining operator of type $M_{a} \times M_{b} \rightarrow M_{c}$ is a linear map $M_{a} \mapsto\left(\operatorname{Hom}\left(M_{b}, M_{c}\right)\right)\{z\}$ satisfying a number of conditions ([FHL93]). The fusion rule $N_{M_{a}, M_{b}}^{M_{c}}$ is the dimension of the space of all intertwining operators of type $M_{a} \times M_{b} \rightarrow M_{c}$. Since $V$ is $C_{2}$-cofinite, the fusion rules are finite ([ABD04]). By the definition of the fusion rules, $N_{M_{a}, M_{b}}^{M_{c}}=N_{M_{a}^{\prime}, M_{b}^{\prime}}^{M^{\prime}}$ if $M_{x} \cong M_{x}^{\prime}$ as $V$-modules for all 
$x=a, b, c$. Hence, the fusion rules are determined by the isomorphism classes of $V$-modules. For irreducible modules $M_{a}$ and $M_{b}$, we use the following expression

$$
\left[M_{a}\right] \times\left[M_{b}\right]=\sum_{[M] \in R(V)} N_{M_{a}, M_{b}}^{M}[M]
$$

which is also called the fusion rule.

Let $M=\left(M, Y_{M}\right)$ be a module for a VOA $V$. For $g \in \operatorname{Aut}(V)$, let $g \circ M=\left(M, Y_{g \circ M}\right)$ denote the $V$-module defined by $Y_{g \circ M}(v, z)=Y_{M}\left(g^{-1} v, z\right)$.

Lemma 1.2. Let $M$ and $M^{\prime}$ be $V$-modules and let $g \in \operatorname{Aut}(V)$.

(1) If $M \cong M^{\prime}$ as $V$-modules then $g \circ M \cong g \circ M^{\prime}$ as $V$-modules.

(2) If $M$ is $\left(h+\mathbb{Z}_{\geq 0}\right)$-graded then so is $g \circ M$.

Proof. Let $f: M \rightarrow M^{\prime}$ be an isomorphism of $V$-modules, that is, $f Y_{M}(v, z) w=Y_{M^{\prime}}(v, z) f(w)$ for all $v \in V$ and $w \in M$. Replacing $v$ by $g^{-1} v$, we obtain $f Y_{g \circ M}(v, z) w=Y_{g \circ M^{\prime}}(v, z) f(w)$ for all $v \in V$. Hence $g \circ M \cong g \circ M^{\prime}$ as $V$-modules, and we obtain (1).

By the axioms of modules, $\omega_{1}$ acts by $m$ on $M_{m}$. Hence (2) follows from $g^{-1}(\omega)=\omega$.

If $M$ is irreducible then so is $g \circ M$. By the lemma above, $\operatorname{Aut}(V)$ acts on $R(V)$. For $\mathcal{T}=\left\{W^{i} \mid 1 \leq i \leq s\right\} \subset R(V)$, let $g \circ \mathcal{T}$ denote $\left\{g \circ W^{i} \mid 1 \leq i \leq s\right\}$.

Lemma 1.3. Let $V$ be a simple rational $C_{2}$-cofinite $V O A$ and let $M_{a}, M_{b}, M_{c}$ be $V$-modules. Let $g \in \operatorname{Aut}(V)$. Then $N_{g \circ M_{a}, g \circ M_{b}}^{g \circ M_{c}}=N_{M_{a}, M_{b}}^{M_{c}}$. In particular, the action of $\operatorname{Aut}(V)$ on $R(V)$ preserves the fusion rules, that is, for $\left[M_{a}\right],\left[M_{b}\right] \in R(V)$,

$$
\left(g \circ\left[M_{a}\right]\right) \times\left(g \circ\left[M_{b}\right]\right)=\sum_{[M] \in R(V)} N_{M_{a}, M_{b}}^{M}(g \circ[M]) .
$$

Proof. By the definition of $g \circ M_{x}$, for each $x \in\{a, b, c\}$, there exists a non-zero linear isomorphism $\nu_{x}: g \circ M_{x} \rightarrow M_{x}$ such that $\nu_{x} Y_{g \circ M_{x}}(v, z)=Y_{M_{x}}\left(g^{-1} v, z\right) \nu_{x}$ for $v \in V$. Let $\mathcal{Y}$ be a non-zero intertwining operator of type $M_{a} \times M_{b} \rightarrow M_{c}$. Then one can verify that

$$
(g \circ \mathcal{Y})(v, z)=\nu_{c}^{-1} \mathcal{Y}\left(\nu_{a} v, z\right) \nu_{b} \in\left(\operatorname{Hom}\left(g \circ M_{b}, g \circ M_{c}\right)\right)\{z\}, v \in g \circ M_{a}
$$

is a non-zero intertwining operator of type $\left(g \circ M_{a}\right) \times\left(g \circ M_{b}\right) \rightarrow\left(g \circ M_{c}\right)$. Hence $N_{M_{a}, M_{b}}^{M_{c}} \leq$ $N_{g \circ M_{a}, g \circ M_{b}}^{g \circ M_{c}}$. Moreover, $N_{g \circ M_{a}, g \circ M_{b}}^{g \circ M} \leq N_{M_{a}, M_{b}}^{M_{c}}$ follows from $g^{-1} \circ(g \circ M) \cong M$. Thus $N_{M_{a}, M_{b}}^{M_{c}}=$ $N_{g \circ M_{a}, g \circ M_{b}}^{g \circ M_{c}}$.

The theory of tensor products of VOAs was established in [FHL93. For a positive integer $k$, let $V^{k}$ denote the tensor product of $k$ copies of $V$. Later, we use the following lemma.

Lemma 1.4. ([FHL93, Section 4.7], [DMZ94]) Let $V$ be a simple rational $C_{2}$-cofinite VOA of CFT type. Then

$$
R\left(V^{k}\right)=\left\{\otimes_{i=1}^{k} W_{i} \mid W_{i} \in R(V)\right\},
$$

and for $\otimes_{i=1}^{k} W_{i, a}, \otimes_{i=1}^{k} W_{i, b}, \otimes_{i=1}^{k} W_{i, c} \in R\left(V^{k}\right)$, the following fusion rule holds:

$$
\otimes_{i=1}^{k} W_{i, a} \times \otimes_{i=1}^{k} W_{i, b}=\otimes_{i=1}^{k}\left(W_{i, a} \times W_{i, b}\right) .
$$




\subsection{Simple current extensions of VOAs}

In this subsection, we recall the notion of simple current modules and simple current extensions.

Let $V(0)$ be a simple VOA. An irreducible $V(0)$-module $M_{a}$ is called a simple current module if for any irreducible $V(0)$-module $M_{b}$, there exists a unique irreducible $V(0)$-module $M_{c}$ satisfying the fusion rule $\left[M_{a}\right] \times\left[M_{b}\right]=\left[M_{c}\right]$. A simple VOA $V$ is called a simple current extension of $V(0)$ graded by a finite abelian group $E$ if $V$ is the direct sum of non-isomorphic irreducible simple current $V(0)$-modules $\{V(\alpha) \mid \alpha \in E\}$ and the fusion rule $[V(\alpha)] \times[V(\beta)]=$ $[V(\alpha+\beta)]$ holds for all $\alpha, \beta \in E$. The uniqueness theorem for simple current extensions was proved in [DM04].

Proposition 1.5. [DM04, Proposition 4.3] Let $(V, Y)$ be a simple current extension of a simple $V O A V(0)$. Then the VOA structure of $V$ as a simple current extension of $V(0)$ is uniquely determined by the $V(0)$-module structure of $V$, that is, if $\left(V^{\prime}, Y^{\prime}\right)$ has a VOA structure with $V=V^{\prime}$ as vector spaces and $Y(v, z)=Y^{\prime}(v, z)$ for all $v \in V(0)$ then the $V O A s(V, Y)$ and $\left(V^{\prime}, Y^{\prime}\right)$ are isomorphic.

The conjugates of a simple current extension by automorphisms were studied in [SY03].

Lemma 1.6. [SY03, Lemma 3.14] Let $V=\oplus_{\alpha \in E} V(\alpha)$ be a simple current extension of a simple VOA $V(0)$ graded by a finite abelian group $E$. Let $g$ be an automorphism of $V(0)$. Then the $V(0)$-module $\oplus_{\alpha \in E} g \circ V(\alpha)$ has a VOA structure isomorphic to $V$.

Let $V=\oplus_{\alpha \in E} V(\alpha)$ be a simple current extension of a simple VOA $V(0)$ graded by a finite abelian group $E$. Then the dual $E^{*}$ of $E$ acts on $V$ as an automorphism group: $\chi \in E^{*}$ acts on $V(\alpha)$ by the scalar multiplication $\chi(\alpha)$ for each $\alpha \in E$. The following restriction homomorphism was studied in [Sh04]:

$$
\eta: N_{\operatorname{Aut}(V)}\left(E^{*}\right) \rightarrow\{g \in \operatorname{Aut}(V(0)) \mid g \circ\{[V(\alpha)] \mid \alpha \in E\}=\{[V(\alpha)] \mid \alpha \in E\}\} .
$$

Proposition 1.7. [Sh04, Theorem 3.3] The map $\eta$ is surjective, and Ker $\eta=E^{*}$.

Let us discuss the group structure on a subset of $R(V)$ under the fusion rules.

Lemma 1.8. (cf. [LY08, Corollary $1(3)]$ ) Let $V$ be a simple rational $C_{2}$-cofinite VOA of CFT type and $\mathcal{T}$ a subset of $R(V)$. Assume that a representative of any element in $\mathcal{T}$ is a self-dual simple current module and that $\mathcal{T}$ is closed under the fusion rules, that is, $W \times W^{\prime}=W^{\prime \prime} \in \mathcal{T}$ for all $W, W^{\prime} \in \mathcal{T}$. Then $(\mathcal{T}, \times)$ is an elementary abelian 2-group.

Proof. By the assumption on $\mathcal{T}$, the fusion rule $\times$ is a binary operation on $\mathcal{T}$. By the assumptions on $V$, the fusion rules are associative $([\mathrm{Hu} 05])$. For any $[M] \in \mathcal{T},[M] \times[M]=[V]$ since $[M]$ is self-dual. Hence $[V] \in \mathcal{T}$, and clearly $[V]$ is the identity. Thus $(\mathcal{T}, \times)$ is an elementary abelian 2-group.

The existence theorem for a simple VOA structure on a direct sum of non-isomorphic selfdual simple current modules was established in [Hu96, LY08]. For the definition of invariant bilinear forms, see [FHL93].

Proposition 1.9. ([Hu96], [LY08, Proposition 3, Theorem 2]) (cf. [Mi04]) Let $V$ be a simple rational $C_{2}$-cofinite $V O A$ of $C F T$ type and let $\mathcal{T}$ be a set of the isomorphism classes of nonisomorphic self-dual $\mathbb{Z}$-graded simple current $V$-modules. Assume that for any element $[M] \in \mathcal{T}$ the invariant bilinear form on $M$ is symmetric. Then $V$-module $\oplus_{[M] \in \mathcal{T}} M$ has a simple VOA structure by extending its $V$-module structure if and only if $\mathcal{T}$ is closed under the fusion rules. 


\subsection{VOA $V_{\sqrt{2} E_{8}}^{+}$}

Let $E_{8}$ denote the $E_{8}$ root lattice and set $\sqrt{2} E_{8}=\left\{\sqrt{2} v \mid v \in E_{8}\right\}$. Let $V_{\sqrt{2} E_{8}}$ be the lattice VOA associated with $\sqrt{2} E_{8}$ ([Bo86, [FLM88]) and let $V_{\sqrt{2} E_{8}}^{+}$be the subspace of $V_{\sqrt{2} E_{8}}$ fixed by the automorphism of $V_{\sqrt{2} E_{8}}$ of order 2 which is a lift of $-1 \in \operatorname{Aut}\left(\sqrt{2} E_{8}\right)$. Then $V_{\sqrt{2} E_{8}}^{+}$is a simple full subVOA of $V_{\sqrt{2} E_{8}}$ of CFT type, and its central charge is 8. Since $\sqrt{2} E_{8}$ has an orthogonal basis of norm $4, V_{\sqrt{2} E_{8}}^{+}$is a framed VOA. By [DGH98], $V_{\sqrt{2} E_{8}}^{+}$is rational and $C_{2}$-cofinite. In this subsection, we review the properties of $V=V_{\sqrt{2} E_{8}}^{+}$and the set $R(V)$ of all isomorphism classes of irreducible $V$-modules.

In AD04, $R(V)$ was determined, and $|R(V)|=2^{10}$. Moreover, in [ADL05, the fusion rules of $R(V)$ were completely calculated and the contragredient modules of irreducible $V$-modules were determined. In particular, any irreducible $V$-module is a self-dual simple current module. By Lemma 1.8, $R(V)$ has an elementary abelian 2-group structure of order $2^{10}$ under the fusion rules. We view $R(V)$ as a 10-dimensional vector space over $\mathbb{F}_{2}$. By Lemma 1.3, Aut $(V)$ acts on $R(V)$ as a subgroup of $\mathrm{GL}(R(V))$.

Let $q_{V}: R(V) \rightarrow \mathbb{F}_{2}$ be the map defined by setting $q_{V}([M])=1$ if $M$ is $(1 / 2+\mathbb{Z})$-graded, and $q_{V}([M])=0$ if $M$ is $\mathbb{Z}$-graded. Note that for $[M] \in R(V)$, the lowest weight of $M$ is $w([M]) / 2$, where $w([M])=0,1$ and 2 if $[M]=[V], q([M])=1$, and $q([M])=0$ with $[M] \neq[V]$ respectively (cf. Section 2.1). It was shown in [Sh04, Theorem 3.8] that $q_{V}$ is a non-singular quadratic form on $R(V)$ of plus type. By Lemma $1.2(2)$, Aut $(V)$ preserves $q_{V}$. Hence we obtain a group homomorphism from $\operatorname{Aut}(V)$ to the orthogonal group $O\left(R(V), q_{V}\right)$. In fact, it is an isomorphism by [Sh04, Theorem 4.5] (cf. [Gr98]).

Since $V_{\sqrt{2} E_{8}}=V \oplus V_{\sqrt{2} E_{8}}^{-}$is a VOA, the invariant bilinear form on the irreducible $V$ module $V_{\sqrt{2} E_{8}}^{-}$is symmetric ([FHL93, Proposition 5.3.6]). By Lemma 1.1 (6), for any $\mathbb{Z}$-graded irreducible $V$-module, the invariant bilinear form on it is also symmetric. Since the lowest weight space of any $(\mathbb{Z}+1 / 2)$-graded irreducible $V$-module is one-dimensional, the invariant form must be symmetric.

Lemma 1.10. Let $V=V_{\sqrt{2} E_{8}}^{+}$.

(1) $V$ is simple, rational, $C_{2}$-cofinite, self-dual and of CFT type.

(2) $\left(R(V), q_{V}\right)$ is a non-singular 10-dimensional quadratic space of plus type over $\mathbb{F}_{2}$.

(3) For $[M] \in R(V)$, the lowest weight of $M$ is $w([M]) / 2$.

(4) $\operatorname{Aut}(V) \cong O\left(R(V), q_{V}\right)$.

(5) For any irreducible $V$-module, the invariant bilinear form on it is symmetric.

\section{Direct sum of quadratic spaces}

Let $(R, q)$ be a non-singular $2 m$-dimensional quadratic space of plus type over $\mathbb{F}_{2}$ and let $k$ be a positive integer. Let $R^{k}$ denote the orthogonal direct sum of $k$ copies of $R$. We use the standard expression $\left(a_{1}, a_{2}, \ldots, a_{k}\right)$ for a vector in $R^{k}$. Let $q^{k}: R^{k} \rightarrow \mathbb{F}_{2},\left(a_{1}, a_{2}, \ldots, a_{k}\right) \mapsto \sum_{i=1}^{k} q\left(a_{i}\right)$. Then $\left(R^{k}, q^{k}\right)$ is a non-singular $2 m k$-dimensional quadratic space of plus type over $\mathbb{F}_{2}$. Note that the symmetric group $\mathrm{Sym}_{k}$ of degree $k$ acts naturally on $R^{k}$ as the permutation group on the coordinates. Clearly, $\operatorname{Sym}_{k} \subset O\left(R^{k}, q^{k}\right)$.

In this section, we introduce a map $w^{k}: R^{k} \rightarrow\{0,1, \ldots, 2 k\}$ and study maximal totally singular subspaces $\mathcal{S}$ of $\left(R^{k}, q^{k}\right)$ such that $w^{k}(v) \geq 4$ for all $v \in \mathcal{S} \backslash\{0\}$. 


\subsection{A construction of maximal totally singular subspaces}

In this subsection, we construct a maximal totally singular subspace of $R^{k}$ from a pair of maximal totally singular subspaces of $R$, which is a slight generalization of [LM82] (cf. [Gr]), and describe its stabilizer in $O(R, q)<\operatorname{Sym}_{k}$.

Let $w: R \rightarrow\{0,1,2\}$ be the map defined by

$$
w(a)= \begin{cases}0 & \text { if } a=0 \\ 1 & \text { if } q(a)=1, \\ 2 & \text { if } q(a)=0 \text { and } a \neq 0\end{cases}
$$

and let

$$
w^{k}: R^{k} \rightarrow\{0,1, \ldots, 2 k\}, \quad\left(a_{1}, a_{2}, \ldots, a_{k}\right) \mapsto \sum_{i=1}^{k} w\left(a_{i}\right) .
$$

Since $w(a) \equiv q(a)(\bmod 2)$ for all $a \in R$, we have $w^{k}(v) \equiv q^{k}(v)(\bmod 2)$ for all $v \in R^{k}$. Hence $w^{k}(v) \in 2 \mathbb{Z}$ if and only if $v$ is singular. The following lemma is easy.

Lemma 2.1. Let $v$ be a vector in $\left(R^{k}, q^{k}\right)$.

(1) $w^{k}(v)=1$ if and only if $v=\sigma(a, 0, \ldots, 0)$ for some non-singular vector $a \in R$ and $\sigma \in$ $\mathrm{Sym}_{k}$.

(2) $w^{k}(v)=2$ if and only if $v=\sigma(a, 0, \ldots, 0)$ or $v=\sigma(b, c, 0, \ldots, 0)$ for some non-zero singular vector $a \in R$ or non-singular vectors $b, c \in R$ and $\sigma \in \operatorname{Sym}_{k}$.

Let $\operatorname{Aut}\left(R^{k}, w^{k}\right)$ denote the subgroup of $\operatorname{GL}\left(R^{k}\right)$ preserving $w^{k}$. Then $\operatorname{Aut}\left(R^{k}, w^{k}\right)$ is a subgroup of $O\left(R^{k}, q^{k}\right)$ since $w^{k}(v) \equiv q^{k}(v)(\bmod 2)$ for $v \in R^{k}$.

Proposition 2.2. $\operatorname{Aut}\left(R^{k}, w^{k}\right) \cong O(R, q)<\operatorname{Sym}_{k}$.

Proof. Let $G=\operatorname{Aut}\left(R^{k}, w^{k}\right)$. Clearly, $O(R, q) \curlywedge \operatorname{Sym}_{k}$ acts faithfully on $R^{k}$ and preserves $w^{k}$. Hence $O(R, q)$ ¿ $\operatorname{Sym}_{k} \subset G$.

Set $R_{(i)}=\left\{\left(a_{1}, a_{2}, \ldots, a_{k}\right) \in R^{k} \mid a_{j}=0\right.$ if $\left.j \neq i\right\}$. By Lemma 2.1 (1),

$$
\left\{v \in R^{k} \mid w^{k}(v)=1\right\}=\left\{v \in \cup_{i=1}^{k} R_{(i)} \mid q^{k}(v)=1\right\} .
$$

Hence $G$ preserves $\left\{v \in \cup_{i=1}^{k} R_{(i)} \mid q^{k}(v)=1\right\}$. Moreover, by Lemma 2.1 (2) and (2.1), $G$ also preserves $\left\{v \in \cup_{i=1}^{k} R_{(i)} \mid q^{k}(v)=0\right\}$. Hence $G$ preserves $\cup_{i=1}^{k} R_{(i)}$.

Let $a, b \in R_{(i)}$ with $\langle a, b\rangle=1$ and let $g \in G$. If $g(a) \in R_{(j)}$ then $g(b) \in R_{(j)}$ since $\left\langle R_{(p)}, R_{(q)}\right\rangle=0$ if $p \neq q$. Since $R$ is non-degenerate, for non-zero $a \in R, \operatorname{Span}_{\mathbb{F}_{2}}\{b \in R \mid\langle b, a\rangle=$ $1\}=R$. Hence $G$ permutes $\left\{R_{(i)} \mid i=1,2, \ldots, k\right\}$. It follows from $O\left(R_{(i)}, q^{k}\right) \cong O(R, q)$ that $G \subset O(R, q) \imath \operatorname{Sym}_{k}$.

Now, let us construct maximal totally singular subspaces $\mathcal{S}$ of $R^{k}$ satisfying

$$
w^{k}(v) \geq 4, \quad \forall v \in \mathcal{S} \backslash\{0\}
$$

as a natural generalization of [LM82] (cf. [Gr]). Let $\Phi, \Psi$ be maximal totally singular subspaces of $R$. For $\{i, j\} \subset\{1,2, \ldots, k\}$, set

$$
\Phi_{(i j)}=\left\{\left(a_{1}, a_{2}, \ldots, a_{k}\right) \in R^{k} \mid a_{i}=a_{j} \in \Phi, a_{l}=0 \text { if } l \neq i, j\right\}
$$


and

$$
\Psi_{(12 \ldots k)}=\left\{(b, \ldots, b) \in R^{k} \mid b \in \Psi\right\} .
$$

Set $(\Phi \cap \Psi)_{(1)}=\left\{(a, 0, \ldots, 0) \in R^{k} \mid a \in \Phi \cap \Psi\right\}$ and $\mathcal{S}(\Phi, \Psi ; k)=\operatorname{Span}_{\mathbb{F}_{2}}\left\{\Phi_{(1 i)}, \Psi_{(12 \ldots k)},(\Phi \cap\right.$ $\left.\Psi)_{(1)} \mid 2 \leq i \leq k\right\}$. Then

$$
\mathcal{S}(\Phi, \Psi ; k)=\left\{\left(a_{1}+b, a_{2}+b, \ldots, a_{k}+b\right) \in R^{k} \mid a_{i} \in \Phi, b \in \Psi, \sum_{i=1}^{k} a_{i} \in \Phi \cap \Psi\right\} .
$$

Remark 2.3. If $k$ is odd then $\mathcal{S}(\Phi, \Psi ; k)=\operatorname{Span}_{\mathbb{F}_{2}}\left\{\Phi_{(1 i)}, \Psi_{(12 \ldots k)} \mid 2 \leq i \leq k\right\}$.

Proposition 2.4. (cf. [LM82, p487]) The subspace $\mathcal{S}(\Phi, \Psi ; k)$ of $\left(R^{k}, q^{k}\right)$ is maximal totally singular. Moreover if $k \geq 3$ and $\Phi \cap \Psi=0$ then it satisfies (2.2).

Proof. By the definition, $\Phi_{(12)}, \Phi_{(13)}, \cdots, \Phi_{(1 k)}$ and $\Psi_{(12 \ldots k)}$ are mutually perpendicular, and $\Phi_{(1 i)}$ and $\Psi_{(12 \ldots k)}$ are totally singular. Hence $\mathcal{S}(\Phi, \Psi ; k)$ is totally singular. Let us count the dimension of $\mathcal{S}(\Phi, \Psi ; k)$. Set $U=\left\{\left(a_{1}, a_{2}, \ldots, a_{k}\right) \in R^{k} \mid a_{i} \in \Phi \cap \Psi\right\}$ and $d=\operatorname{dim} \Phi \cap \Psi$. Then $U \subset \mathcal{S}(\Phi, \Psi ; k)$ and $\operatorname{dim} U=k d$. Set $T=\mathcal{S}(\Phi, \Psi ; k) / U$. Then $T=\oplus_{i=2}^{k} \bar{\Phi}_{(1 i)} \oplus \bar{\Psi}_{(12 \ldots k)}$ as vector spaces, where $\bar{\Psi}=\Psi /(\Phi \cap \Psi)$ and $\bar{\Phi}=\Phi /(\Phi \cap \Psi)$. Since this is a direct sum, $\operatorname{dim} T=(k-1) \operatorname{dim} \bar{\Phi}+\operatorname{dim} \bar{\Psi}$. Hence

$$
\operatorname{dim} \mathcal{S}(\Phi, \Psi ; k)=\operatorname{dim} T+\operatorname{dim} U=(k-1)(m-d)+(m-d)+k d=m k .
$$

Thus $\mathcal{S}(\Phi, \Psi ; k)$ is a maximal totally singular subspace of $\left(R^{k}, q^{k}\right)$.

Assume that $k \geq 3$ and that $\Phi \cap \Psi=0$. Let $v=\left(a_{1}+b, a_{2}+b, \ldots, a_{k-1}+b, \sum_{i=1}^{k-1} a_{i}+b\right) \in$ $\mathcal{S}(\Phi, \Psi ; k)$ with $w^{k}(v) \leq 2$, where $a_{i} \in \Phi$ and $b \in \Psi$. Then at least $(k-2)$ entries are zero by Lemma 2.1 (2). Say $a_{i}+b=0,1 \leq i \leq k-2$, which implies $a_{i}=b=0$ by $\Psi \cap \Phi=0$ and $k \geq 3$. Hence $v=\left(0, \ldots, 0, a_{k-1}, a_{k-1}\right)$. Since $a_{k-1}$ is singular and $w^{k}(v) \leq 2$, we obtain $a_{k-1}=0$ by Lemma 2.1 (2). Therefore $v=0$.

Let us describe the stabilizer of $\mathcal{S}(\Phi, \Psi ; k)$ in $\operatorname{Aut}\left(R^{k}, w^{k}\right)$. Let $O(R, q)^{k}$ denote the normal subgroup of $O(R, q) \backslash \mathrm{Sym}_{k}$ isomorphic to the direct product of $k$ copies of the orthogonal group $O(R, q)$. For an element $g \in O(R, q)^{k}$, we use the expression $g=\left(g_{1}, g_{2}, \ldots, g_{k}\right)$, where $g_{i} \in O(R, q)$. For a subgroup $F$ of $O(R, q)$, we denote $F_{(i j)}=\left\{\left(g_{1}, g_{2}, \ldots, g_{k}\right) \in O(R, q)^{k} \mid g_{i}=\right.$ $g_{j} \in F, g_{l}=1$ if $\left.l \neq i, j\right\}$ and $F_{(12 \ldots k)}=\left\{(h, \ldots, h) \in O(R, q)^{k} \mid h \in F\right\}$.

Proposition 2.5. Let $G$ be the stabilizer of $\mathcal{S}(\Phi, \Psi ; k)$ in $\operatorname{Aut}\left(R^{k}, w^{k}\right)$. Let $H$ be the stabilizer of $\Phi$ in $O(R, q)$ and let $K$ be the stabilizer of $\Psi$ in $H$. If $k \geq 3$ and $\Phi \cap \Psi=0$ then $G$ is generated by $\mathrm{O}_{2}(H)_{(1 i)}\left(\cong 2^{\left(\begin{array}{c}m \\ 2\end{array}\right)}\right),(2 \leq i \leq k), K_{(12 \ldots k)}\left(\cong \mathrm{SL}_{m}(2)\right)$ and $\mathrm{Sym}_{k}$, and $G$ has the shape $2^{(k-1)\left(\begin{array}{c}m \\ 2\end{array}\right)}:\left(\mathrm{SL}_{m}(2) \times \operatorname{Sym}_{k}\right)$.

Proof. By Lemma 1.1 (3) and (4), $H=O_{2}(H): K$. By Proposition 2.2, $\operatorname{Aut}\left(R^{k}, w^{k}\right)=$ $O(R, q)$ i $\mathrm{Sym}_{k}$.

Clearly, $\operatorname{Sym}_{k}$ preserves $\mathcal{S}(\Phi, \Psi ; k)$. By Lemma $1.1(3)$ and $(2.3), O_{2}(H)_{(1 i)}(2 \leq i \leq k)$ and $K_{(12 \ldots k)}$ also preserve $\mathcal{S}(\Phi, \Psi ; k)$. Hence $G$ contains $\left\langle O_{2}(H)_{(1 i)}, K_{(12 \ldots k)}, \operatorname{Sym}_{k} \mid 2 \leq i \leq k\right\rangle$.

Let $g \in G$. Up to $\operatorname{Sym}_{k}$, we may assume $g=\left(g_{1}, g_{2}, \ldots, g_{k}\right) \in O(R, q)^{k} \cap G$. If follows from $k \geq 3$ that $g$ preserves $\Phi_{(1 i)}$ for all $i$. Hence $g_{i} \in H$ and $g_{i} \in O_{2}(H) g_{1}$. Up to $K_{(12 \ldots k)}$, we may assume $g_{i} \in O_{2}(H)$ for all $i$ since $H=O_{2}(H): K$. Moreover, up to $\left\langle O_{2}(H)_{(1 i)} \mid 2 \leq i \leq k\right\rangle$, we may assume $g_{i}=1$ if $i \geq 2$. Let $b \in \Psi$. Then $g_{1}(b)=b+c$ for some $c \in \Phi$, and $(b, b, \ldots, b)+g(b, b, \ldots, b)=(c, 0, \ldots, 0) \in \mathcal{S}(\Phi, \Psi ; k)$. Since $\Phi \cap \Psi=0$, we have $c=0$ by (2.3), and $g_{1}=1$ by Lemma $1.1(3)$. Thus $g \in\left\langle O_{2}(H)_{(1 i)}, K_{(12 \ldots k)}, \operatorname{Sym}_{k} \mid 2 \leq i \leq k\right\rangle$.

One can easily see that $G$ has the desired shape by Lemma 1.1 (3) and (4).

Later, we need the following lemma. 
Lemma 2.6. Let $v$ be a vector in $\mathcal{S}(\Phi, \Psi ; 3)$ with $w^{3}(v)=4$. Assume that $\Phi \cap \Psi=0$. Then one of the following holds:

(I) $v=\sigma(a, a, 0)$ for some $a \in \Phi \backslash\{0\}$ and $\sigma \in \mathrm{Sym}_{3}$.

(II) $v=\sigma(a+c, a+b+c, b+c)$ for some $\sigma \in \mathrm{Sym}_{3}, a, b \in \Phi$ and $c \in \Psi \backslash\{0\}$ satisfying $w(a+c)=2$ and $w(a+b+c)=w(b+c)=1$.

Moreover, the numbers of vectors of type (I) and (II) are $3 \times\left(2^{m}-1\right)$ and $3 \times\left(2^{m}-1\right) \times 2^{2 m-2}$ respectively.

Proof. The first assertion follows from (2.3). The number of vectors of type (I) can be calculated by $|\Phi \backslash\{0\}|=2^{m}-1$. Let us count the number of vectors of type (II). Note that $w(a+c)=2$ and $w(a+b+c)=w(b+c)=1$ if and only if $\langle a, c\rangle=0$ and $\langle b, c\rangle=1$. Clearly, there are $2^{m}-1$ choices of $c \in \Psi \backslash\{0\}$. For each $c$, there are $2^{m-1}$ choices of $a$ since $\{u \in \Phi \mid\langle u, c\rangle=0\}$ is an $(m-1)$-dimensional subspace of $\Phi$. Similarly, there are $2^{m-1}$ choices of $b$ since $|\{u \in \Phi \mid\langle u, c\rangle=1\}|=2^{m}-2^{m-1}=2^{m-1}$. Hence the number of vectors of type (II) is $3 \times\left(2^{m}-1\right) \times 2^{2 m-2}$.

\subsection{Uniqueness of maximal totally singular subspaces for $k=3$}

In this subsection, we consider the case $k=3$, and show that any maximal totally singular subspace of $R^{3}$ satisfying (2.2) is conjugate to $\mathcal{S}(\Phi, \Psi ; 3)$ with $\Phi \cap \Psi=0$ under $O(R, q)^{3}$. For the definition of $\mathcal{S}(\Phi, \Psi ; 3)$, see (2.3).

Let $\rho_{i}$ denote the $i$-th coordinate projection from $R^{3}$ to $R$. For a subspace $\mathcal{S}$ of $R^{3}$ and distinct $i, j \in\{1,2,3\}$, we denote $\mathcal{S}^{(i)}=\left\{v \in \mathcal{S} \mid \rho_{i}(v)=0\right\}$ and $\mathcal{S}^{(i j)}=\left\{v \in \mathcal{S} \mid \rho_{i}(v)=\right.$ $\left.\rho_{j}(v)=0\right\}$.

Lemma 2.7. Let $\mathcal{S}$ be a maximal totally singular subspace of $R^{3}$ satisfying (2.2) and let $i, j \in$ $\{1,2,3\}$ such that $i \neq j$. Then the following hold:

(1) $\mathcal{S}^{(i j)}=0$.

(2) $\rho_{i}(\mathcal{S})=R$.

(3) The dimension of $\mathcal{S}^{(i)}$ is $m$.

(4) $\rho_{j}\left(\mathcal{S}^{(i)}\right)$ is a maximal totally singular subspace of $R$.

Proof. Without loss of generality, we may assume that $i=1$ and $j=2$.

Let $v=(0,0, a) \in \mathcal{S}^{(12)}$. Then $v$ must be 0 by (2.2), which shows (1).

Suppose $\rho_{1}(\mathcal{S}) \neq R$. Then $\rho_{1}(\mathcal{S})^{\perp} \neq 0$. Let $a \in \rho_{1}(\mathcal{S})^{\perp} \backslash\{0\}$. Then $(a, 0,0) \in \mathcal{S}^{\perp}=\mathcal{S}$ by Lemma 1.1 (1), which contradicts (1). Hence (2) holds.

By (2), the projection $\rho_{1}: \mathcal{S} \rightarrow R$ is surjective. It follows from $\operatorname{dim} R=2 m$ and $\operatorname{dim} \mathcal{S}=3 m$ that $\operatorname{dim} \mathcal{S}^{(1)}=m$. Hence we have $(3)$.

Let $v=(0, a, b) \in \mathcal{S}^{(1)}$. Since $v$ is singular, both $a$ and $b$ must be singular by (2.2). Hence $\rho_{2}\left(\mathcal{S}^{(1)}\right)$ is totally singular. By $(1)$ and $(3), \operatorname{dim} \rho_{2}\left(\mathcal{S}^{(1)}\right)=m$, and hence $\rho_{2}\left(\mathcal{S}^{(1)}\right)$ is a maximal totally singular subspace of $R$, which proves (4).

Recall that $\operatorname{Aut}\left(R^{3}, w^{3}\right) \cong O(R, q)<\mathrm{Sym}_{3}$ from Proposition 2.2 and that $O(R, q)^{3}$ is the normal subgroup of $\operatorname{Aut}\left(R^{3}, w^{3}\right)$ isomorphic to the direct product of three copies of $O(R, q)$. 
Theorem 2.8. Let $\mathcal{S}$ be a maximal totally singular subspace of $\left(R^{3}, q^{3}\right)$ satisfying (2.2). Then $\mathcal{S}$ is conjugate to $\mathcal{S}(\Phi, \Psi ; 3)$ under $O(R, q)^{3}$ for some maximal totally singular subspaces $\Phi$ and $\Psi$ of $(R, q)$ satisfying $\Phi \cap \Psi=0$. Moreover $O(R, q)^{3}$ is transitive on the set of all maximal totally singular subspaces of $\left(R^{3}, q^{3}\right)$ satisfying (2.2).

Proof. Set $\Phi=\rho_{1}\left(\mathcal{S}^{(3)}\right)$ and $\Phi^{\prime}=\rho_{2}\left(\mathcal{S}^{(3)}\right)$. Then both $\Phi$ and $\Phi^{\prime}$ are maximal totally singular subspaces of $R$ by Lemma 2.7 (4). Up to the action of $O(R, q)$ on the second coordinate, we may assume that $\Phi^{\prime}=\Phi$ by Lemma 1.1 (2). It follows from Lemma 1.1 (3) that $\operatorname{Stab}_{O(R, q)}\left(\Phi^{\prime}\right) / O_{2}\left(\operatorname{Stab}_{O(R, q)}\left(\Phi^{\prime}\right)\right) \cong \operatorname{SL}_{m}(2)$ acts naturally on $\Phi^{\prime}$. Hence we may assume that $\mathcal{S}^{(3)}=\{(a, a, 0) \mid a \in \Phi\}$ up to the action of $O(R, q)$ on the second coordinate. By the same arguments on $\rho_{1}\left(\mathcal{S}^{(2)}\right)$ and $\rho_{3}\left(\mathcal{S}^{(2)}\right)$, we obtain $\mathcal{S}^{(2)}=\left\{(a, 0, a) \mid a \in \Phi^{\prime \prime}\right\}$ for some maximal totally singular subspace $\Phi^{\prime \prime}$ of $R$ up to the action of $O(R, q)$ on the third coordinate. Since $\mathcal{S}$ is totally singular, we have $\left\langle\mathcal{S}^{(3)}, \mathcal{S}^{(2)}\right\rangle=0$, equivalently, $\Phi^{\prime \prime} \subset \Phi^{\perp}$. By Lemmas 1.1 (1) and 2.7 (4), $\Phi^{\prime \prime}=\Phi$. Hence we obtain two $m$-dimensional totally singular subspaces $\Phi_{(12)}=\{(a, a, 0) \mid a \in \Phi\}$ and $\Phi_{(13)}=\{(a, 0, a) \mid a \in \Phi\}$ of $\mathcal{S}$.

Let $c \in R \backslash \Phi$. Then by Lemma $2.7(2)$, there exist $a, b \in R$ such that $v=(a, b, c) \in \mathcal{S}$. It follows from $\left\langle\Phi_{(13)}, v\right\rangle=0$ that $\langle p, a+\Phi\rangle=\langle p, c+\Phi\rangle$ for all $p \in \Phi$. Note that $\{\langle p, \cdot\rangle \mid p \in$ $\Phi\}=\operatorname{Hom}\left(R / \Phi, \mathbb{F}_{2}\right)$ by Lemma 1.1 (1). Hence $a \in c+\Phi$. Similarly, $a \in b+\Phi$. Hence there exist $u \in \Phi_{(12)}$ and $x(c) \in \Phi$ such that $v+u=(x(c)+c, c, c) \in \mathcal{S}$. By (2.2) $x(c)$ is uniquely determined by $c$. Hence for $c_{1}, c_{2} \in R \backslash \Phi$ with $c_{1}+c_{2} \notin \Phi$ we have $x\left(c_{1}\right)+x\left(c_{2}\right)=x\left(c_{1}+c_{2}\right)$.

Let $\Psi$ be a maximal totally singular subspace of $R$ such that $\Phi \cap \Psi=0$. Set $\mathcal{T}=\{(x(c)+$ $c, c, c) \mid c \in \Psi\}$. Then $\mathcal{T}$ is an $m$-dimensional totally singular subspace of $\mathcal{S}$. Since $\rho_{2}(\mathcal{T})=$ $\rho_{3}(\mathcal{T})=\Psi$ is totally singular, so is $\rho_{1}(\mathcal{T})$. It follows from $\Phi \cap \Psi=0$ that $\Phi \cap \rho_{1}(\mathcal{T})=0$. By Lemma $1.1(5), \rho_{1}(\mathcal{T})$ is conjugate to $\Psi$ under $O(R, q)$. Hence we may assume $x(c)=0$ for all $c \in \Psi$ up to the action of $O(R, q)$ on the first coordinate. Then $\mathcal{T}=\Psi_{(123)}=\{(c, c, c) \mid c \in \Psi\}$. Hence $\mathcal{S}$ is conjugate to $\mathcal{S}(\Phi, \Psi ; 3)$ under $O(R, q)^{3}$.

The transitivity of $O(R, q)^{3}$ follows from Lemma 1.1 (5).

\section{Application to binary codes and lattices}

In this section, we apply the results in the previous section to binary codes and lattices. For the definitions and fundamental facts on binary codes and lattices, we refer the reader to [MS77, CS99].

\subsection{Application to binary codes}

In this subsection, we construct doubly even self-dual binary codes without codewords of weight 4, which is a slight generalization of Turyn's construction of the extended binary Golay code (cf. [MS77, Ch 18, Section 7.4]).

Let $($,$) be the standard inner product on \mathbb{F}_{2}^{n}$. For $x=\left(x_{1}, x_{2}, \ldots, x_{n}\right) \in \mathbb{F}_{2}^{n}$, wt $(x)=\mid\{i \mid$ $\left.x_{i} \neq 0\right\} \mid$ is the (Hamming) weight of $x$. For $x, y \in \mathbb{F}_{2}^{n}$, the following holds:

$$
(x, y) \equiv \frac{1}{2}(\mathrm{wt}(x+y)-\mathrm{wt}(x)-\mathrm{wt}(y)) \quad(\bmod 2)
$$

A subset of $\mathbb{F}_{2}^{n}$ is called a binary (linear) code of length $n$ if it is a subspace. Let $C^{\perp}$ denote the dual code of a binary code $C$ of length $n$, that is, $C^{\perp}=\left\{c \in \mathbb{F}_{2}^{n} \mid(c, C)=0\right\}$. A binary code $C$ is said to be doubly even if $\operatorname{wt}(c) \in 4 \mathbb{Z}$ for all $c \in C$, and is said to be self-dual if $C=C^{\perp}$. We now consider the following condition on $C^{\perp}$ :

$$
\mathrm{wt}(c) \in 2 \mathbb{Z} \quad \forall c \in C^{\perp} .
$$


Note that $C^{\perp}$ satisfies (3.2) if and only if $C$ contains the all-one codeword $\mathbf{1}_{n}$.

Let $C$ be a doubly even binary code of length $n \in 8 \mathbb{Z}$ satisfying (3.2). Set $R(C)=C^{\perp} / C^{2}$ and let

$$
\varphi_{C}: C^{\perp} \rightarrow R(C), \quad c \mapsto c+C
$$

be the canonical map. Then $R(C) \cong(\mathbb{Z} / 2 \mathbb{Z})^{m}$ for some $m \in \mathbb{Z}_{>0}$ and we view it as a vector space over $\mathbb{F}_{2}$. Note that $\operatorname{dim} C=(n-m) / 2$. Let $\langle$,$\rangle be the symmetric bilinear form on R(C)$ defined by $\langle c+C, d+C\rangle=(c, d)$. By (3.2) and $\left(C^{\perp}\right)^{\perp}=C$, it is a non-singular symplectic form. Consider the map

$$
q_{C}: R(C) \rightarrow \mathbb{F}_{2}, \quad x+C \mapsto \operatorname{wt}(x) / 2 \quad(\bmod 2) .
$$

It follows from $\operatorname{wt}(c) \in 4 \mathbb{Z}$ for all $c \in C,\left(C, C^{\perp}\right)=0$ and (3.1) that $q_{C}$ is a well-defined quadratic form on $R(C)$ associated to the symplectic form $\langle$,$\rangle . Since n \in 8 \mathbb{Z}$, the type of $q_{C}$ is plus. Thus $\left(R(C), q_{C}\right)$ is a non-singular $m$-dimensional quadratic space of plus type over $\mathbb{F}_{2}$.

Let $\mathcal{S}$ be a maximal totally singular subspace of $\left(R(C)^{k}, q_{C}^{k}\right)$. Let $\mathfrak{C}(\mathcal{S})$ be the inverse image of $\mathcal{S}$ with respect to the canonical map

$$
\varphi_{C}^{k}:\left(C^{\perp}\right)^{k} \rightarrow R(C)^{k}, \quad\left(c_{i}\right) \mapsto\left(\varphi_{C}\left(c_{i}\right)\right),
$$

that is,

$$
\mathfrak{C}(\mathcal{S})=\left(\varphi_{C}^{k}\right)^{-1}(\mathcal{S})
$$

Then $\mathfrak{C}(\mathcal{S})$ is a binary code of length $n k$. Note that $\operatorname{Ker} \varphi_{C}^{k}=C^{k}$, where $C^{k}$ is the direct sum of $k$ copies of $C$, and that $\operatorname{dim} \operatorname{Ker} \varphi_{C}^{k}=(n-m) k / 2$.

Theorem 3.1. Let $C$ be a doubly even binary code of length $n \in 8 \mathbb{Z}$ satisfying (3.2) and let $\mathcal{S}$ be a maximal totally singular subspace of $\left(R(C)^{k}, q_{C}^{k}\right)$.

(1) The binary code $\mathfrak{C}(\mathcal{S})$ of length $n k$ is doubly even and self-dual.

(2) Assume that $\mathcal{S}$ satisfies (2.2). If $C$ has no codewords of weight 4 then so does $\mathfrak{C}(\mathcal{S})$.

Proof. Since $\mathcal{S}$ is totally singular, $\mathfrak{C}(\mathcal{S})$ is doubly even. It follows from the maximality of $\mathcal{S}$ that $\operatorname{dim} \mathcal{S}=m k / 2$. Hence

$$
\operatorname{dim} \mathfrak{C}(\mathcal{S})=\operatorname{dim} \operatorname{Ker} \varphi_{C}^{k}+\operatorname{dim} \mathcal{S}=k(n-m) / 2+m k / 2=k n / 2,
$$

which shows that $\mathfrak{C}(\mathcal{S})$ is self-dual.

By the definition of the map $w: R(C) \rightarrow\{0,1,2\}$ (cf. Section 2.1), the minimum weight of $c+C \in R(C)$ is greater than or equal to $2 w(c+C)$. Hence for $u \in R(C)^{k}$ the minimum weight of $\left(\varphi_{C}^{k}\right)^{-1}(u)$ is greater than or equal to $2 w^{k}(u)$. Thus (2) follows from (2.2) and the assumption on $C$.

Remark 3.2. In general, the minimum weight of $c+C \in R(C)$ may not be equal to $2 w(c+C)$.

Remark 3.3. Let $C$ be a doubly even binary code of length $n \in 8 \mathbb{Z}$ satisfying (3.2). Let $A$ and $B$ be doubly even self-dual binary codes of length $n$ satisfying $A \cap B \supset C$. Then $\Phi=\varphi_{C}(A)$ and $\Psi=\varphi_{C}(B)$ are maximal totally singular subspaces of $R(C)$. By (2.3) $\mathfrak{C}(\mathcal{S}(\Phi, \Psi ; k))$ is described in terms of $A$ and $B$ as follows:

$$
\mathfrak{C}(\mathcal{S}(\Phi, \Psi ; k))=\left\{\left(a_{1}+b, a_{2}+b, \ldots, a_{k}+b\right) \in \mathbb{F}_{2}^{n k} \mid a_{i} \in A, b \in B, \sum_{i=1}^{k} a_{i} \in A \cap B\right\}
$$




\subsection{Turyn's construction of the extended binary Golay code}

In this subsection, we consider the case where $k=3$ and $C=\operatorname{Span}_{\mathbb{F}_{2}}\left\{\mathbf{1}_{8}\right\}$, and $\operatorname{describe}$ the extended binary Golay code of length 24. Moreover, as an application of Section 2.2, we study the extended binary Golay code and the Mathieu group. We continue the notation of the previous subsection.

Obviously, $C$ has no codewords of weight 4 and satisfies (3.2). Let $\mathcal{S}$ be a maximal totally singular subspace of $\left(R(C)^{3}, q_{C}^{3}\right)$ satisfying (2.2). Note that such a subspace exists by Proposition 2.4. By Theorem 3.1, $\mathfrak{C}(\mathcal{S})$ is a doubly even self-dual binary code of length 24 without codewords of weight 4 . It is well-known that such a binary code is equivalent to the extended binary Golay code of length 24 (cf. [MS77]).

Corollary 3.4. Let $C=\operatorname{Span}_{\mathbb{F}_{2}}\left\{\mathbf{1}_{8}\right\}$ and let $\mathcal{S}$ be a maximal totally singular subspace of $\left(R(C)^{3}, q_{C}^{3}\right)$ satisfying (2.2). Then $\mathfrak{C}(\mathcal{S})$ is equivalent to the extended binary Golay code of length 24 .

Remark 3.5. Let $\Phi$ and $\Psi$ be maximal totally singular subspaces of $\left(R(C), q_{C}\right)$ satisfying $\Phi \cap \Psi=0$. Then $\mathfrak{C}(\mathcal{S}(\Phi, \Psi ; 3))$ is isomorphic to the extended binary Golay code, which is called Turyn's construction (cf. [MS77, Ch 18, Theorem 12]). Note that $\varphi_{C}^{-1}(\Phi)$ and $\varphi_{C}^{-1}(\Psi)$ are isomorphic to the extended Hamming code of length 8.

Since $\operatorname{Aut}\left(C^{\perp}\right)=\operatorname{Aut}(C)$, there is a canonical homomorphism of groups

$$
\psi_{C}: \operatorname{Aut}\left(C^{\perp}\right) \rightarrow O\left(R(C), q_{C}\right) .
$$

The injectivity is clear, and the surjectivity follows from $\operatorname{Aut}(C)=\operatorname{Sym}_{8} \cong O\left(R(C), q_{C}\right)$. Hence $\psi_{C}$ is an isomorphism of groups.

A subcode of $\mathfrak{C}(\mathcal{S})$ is called a trio if it is equivalent to $C^{3}=C \oplus C \oplus C$. Since $\operatorname{Aut}\left(C^{3}\right) \cong$ $\operatorname{Aut}(C) \gtrless \operatorname{Sym}_{3}$ and $\operatorname{Aut}\left(C^{3}\right)=\operatorname{Aut}\left(\left(C^{\perp}\right)^{3}\right), \psi_{C}$ induces the injective homomorphism of groups

$$
\psi_{C}^{3}: \operatorname{Aut}\left(\left(C^{\perp}\right)^{3}\right) \rightarrow \operatorname{Aut}\left(R(C)^{3}, w^{3}\right) .
$$

By Proposition 2.2, $\psi_{C}^{3}$ is an isomorphism. Note that $\psi_{C}^{3}$ is compatible with $\varphi_{C}^{3}$, that is, $\varphi_{C}^{3}(g(c))=\psi_{C}^{3}(g)\left(\varphi_{C}^{3}(c)\right)$ for $g \in \operatorname{Aut}\left(\left(C^{\perp}\right)^{3}\right)$ and $c \in\left(C^{\perp}\right)^{3}$.

As corollaries of Proposition 2.5 and Theorem [2.8, we obtain the following well-known properties of $\operatorname{Aut}(\mathfrak{C}(\mathcal{S}))$.

Corollary 3.6. (cf. CS99]) Let $C=\operatorname{Span}_{\mathbb{F}_{2}}\left\{\mathbf{1}_{8}\right\}$ and let $\mathcal{S}$ be a maximal totally singular subspace of $\left(R(C)^{3}, q_{C}^{3}\right)$ satisfying (2.2). Let $G$ be the stabilizer of $C^{3}$ in the automorphism group of $\mathfrak{C}(\mathcal{S})$.

(1) The automorphism group of $\mathfrak{C}(\mathcal{S})$ is transitive on the set of all trios of $\mathfrak{C}(\mathcal{S})$.

(2) $G$ is isomorphic to the stabilizer of $\mathcal{S}$ in $\operatorname{Aut}\left(R(C)^{3}, w^{3}\right)$, and it has the shape $2^{6}:\left(\mathrm{SL}_{3}(2) \times\right.$ $\mathrm{Sym}_{3}$ ).

Proof. Let $D$ be a trio of $\mathfrak{C}(\mathcal{S})$. It suffices to show that there is an automorphism of $\mathfrak{C}(\mathcal{S})$ which sends $D$ to $C^{3}$. Let $g \in \operatorname{Sym}_{24}$ such that $g(D)=C^{3}$. Since $g(\mathfrak{C}(\mathcal{S}))$ is a doubly even self-dual binary code without codewords of weight 4 , its image $\varphi_{C}^{3}(g(\mathfrak{C}(\mathcal{S})))$ is a maximal totally singular subspace of $R(C)^{3}$ satisfying (2.2). Then by Theorem 2.8, there exists $h \in \operatorname{Aut}\left(C^{3}\right)(\cong$ $\left.\operatorname{Aut}\left(R(C)^{3}, w^{3}\right)\right)$ such that $h\left(C^{3}\right)=C^{3}$ and $h(g(\mathfrak{C}(\mathcal{S})))=\mathfrak{C}(\mathcal{S})$, which proves $(1)$.

Clearly, $G$ is equal to the stabilizer of $\mathfrak{C}(\mathcal{S})$ in $\operatorname{Aut}\left(\left(C^{\perp}\right)^{3}\right)$. Since $\psi_{C}^{3}$ is compatible with $\varphi_{C}^{3}$ and $\varphi_{C}^{3}(\mathfrak{C}(\mathcal{S}))=\mathcal{S}, \psi_{C}^{3}(G)$ is the stabilizer of $\mathcal{S}$ in $\operatorname{Aut}\left(R(C)^{3}, w^{3}\right)$. Since $\psi_{C}^{3}$ is an isomorphism, 
$G$ is isomorphic to the stabilizer of $\mathcal{S}$ in $\operatorname{Aut}\left(R(C)^{3}, w^{3}\right)$. Moreover, $G$ is isomorphic to the stabilizer of $\mathcal{S}(\Phi, \Psi ; 3)$ in $\operatorname{Aut}\left(R(C)^{3}, w^{3}\right)$ by $(1)$. Hence we obtain (2) by Proposition 2.5.

Let us count the number of codewords of weight 8 in $\mathfrak{C}(\mathcal{S})$. The following lemma is easy.

Lemma 3.7. Let $u \in R(C)$.

(1) If $w(u)=0$ then the number of codewords of weight 8 in $\varphi_{C}^{-1}(u)$ is 1.

(2) If $w(u)=1$ then the number of codewords of weight 2 in $\varphi_{C}^{-1}(u)$ is 1 .

(3) If $w(u)=2$ then the number of codewords of weight 4 in $\varphi_{C}^{-1}(u)$ is 2 .

Proposition 3.8. Let $C=\operatorname{Span}_{\mathbb{F}_{2}}\left\{\mathbf{1}_{8}\right\}$ and let $\mathcal{S}$ be a maximal totally singular subspace of $\left(R(C)^{3}, q_{C}^{3}\right)$ satisfying (2.2). Then the number of codewords of weight 8 in $\mathfrak{C}(\mathcal{S})$ is 759 .

Proof. By Theorem 2.8 and the surjectivity of $\psi_{C}^{3}$, we may assume that $\mathcal{S}=\mathcal{S}(\Phi, \Psi ; 3)$. Any codeword of weight 8 in $\mathfrak{C}(\mathcal{S})$ belongs to $\left(\varphi_{C}^{3}\right)^{-1}(0)$ or $\left(\varphi_{C}^{3}\right)^{-1}(u)$ for some $u \in R(C)^{3}$ with $w^{3}(u)=4$. Note that $\left(\varphi_{C}^{3}\right)^{-1}(0)=C^{3}$. Hence by Lemmas 2.6 and 3.7, the number of codewords of weight 8 in $\mathfrak{C}(\mathcal{S})$ is

$$
(3 \times 1)+\left(3 \times\left(2^{3}-1\right)\right) \times 1^{0} \times 2^{2}+\left(3 \times\left(2^{3}-1\right) \times 2^{4}\right) \times 1^{2} \times 2^{1}=759 .
$$

\subsection{Application to lattices}

In this subsection, we construct even unimodular lattices without vectors of norm 2, which was studied by Griess [Gr].

Let $\left(\right.$, ) be a positive definite symmetric bilinear form on $\mathbb{R}^{n}$. For $x \in \mathbb{R}^{n},(x, x)$ is the (squared) norm of $x$. For $x, y \in \mathbb{R}^{n}$, the following holds:

$$
2(x, y)=(x+y, x+y)-(x, x)-(y, y)
$$

A subset $L$ of $\mathbb{R}^{n}$ is called a lattice of rank $n$ if $L$ has a basis $e_{1}, e_{2}, \ldots, e_{n}$ of $\mathbb{R}^{n}$ satisfying $L=\oplus_{i=1}^{n} \mathbb{Z} e_{i}$. Let $L^{*}$ denote the dual lattice of a lattice $L$ of rank $n$, that is, $L^{*}=\left\{v \in \mathbb{R}^{n} \mid\right.$ $(v, L) \subset \mathbb{Z}\}$. A lattice $L$ is said to be even if $(v, v) \in 2 \mathbb{Z}$ for all $v \in L$, and is said to be unimodular if $L=L^{*}$. We now consider the following condition on $L^{*}$ :

$$
(v, v) \in \mathbb{Z} \quad \forall v \in L^{*}
$$

Lemma 3.9. Let $L$ be an even lattice of rank $n \in 8 \mathbb{Z}$.

(1) L satisfies (3.4) if and only if $L$ contains $\sqrt{2} J$ for some even unimodular lattice $J$.

(2) If L satisfies (3.4) then $2 L^{*} \subset L$.

Proof. If $L \supset \sqrt{2} J$ for some even unimodular lattice $J$ then $L^{*} \subset J / \sqrt{2}$. Since $(v, v) \in \mathbb{Z}$ for any $v \in J / \sqrt{2}, L^{*}$ satisfies (3.4).

Conversely, we assume that $L$ satisfies (3.4). Then $\sqrt{2} L^{*}$ is even. Since $n \in 8 \mathbb{Z}$, there exists an even unimodular lattice $J$ of rank $n$ such that $\sqrt{2} L^{*} \subset J$ (cf. [Ve79]). Hence $J \subset\left(\sqrt{2} L^{*}\right)^{*}=$ $L / \sqrt{2}$, equivalently, $\sqrt{2} J \subset L$, and obtain (1).

If $L$ satisfies (3.4) then $\sqrt{2} L^{*} \subset\left(\sqrt{2} L^{*}\right)^{*}=L / \sqrt{2}$, and obtain (2). 
Let $L$ be an even lattice of rank $n \in 8 \mathbb{Z}$ satisfying (3.4). Set $R(L)=L^{*} / L$ and let

$$
\varphi_{L}: L^{*} \rightarrow R(L), \quad v \mapsto v+L
$$

be the canonical map. Then by Lemma $3.9(2), R(L) \cong(\mathbb{Z} / 2 \mathbb{Z})^{m}$ for some $m \in \mathbb{Z}_{\geq 0}$ and we view it as a vector space over $\mathbb{F}_{2}$. Note that the determinant of $L$ is $2^{m / 2}$. Let $\langle$,$\rangle be the$ symmetric bilinear form on $R(L)$ defined by $\langle v+L, u+L\rangle=2(v, u)(\bmod 2)$. By (3.4) and $\left(L^{*}\right)^{*}=L$, it is a non-singular symplectic form. Consider the map

$$
q_{L}: R(L) \rightarrow \mathbb{F}_{2}, \quad v+L \mapsto(v, v) \quad(\bmod 2)
$$

It follows from $(v, v) \in 2 \mathbb{Z}$ for all $v \in L,\left(L, L^{*}\right) \subset \mathbb{Z}$ and (3.3) that $q_{L}$ is a well-defined quadratic form on $R(L)$ associated to $\langle$,$\rangle . Since n \in 8 \mathbb{Z}$, the type of $q_{L}$ is plus (cf. [Ve79]). Thus $\left(R(L), q_{L}\right)$ is a non-singular $m$-dimensional quadratic space of plus type over $\mathbb{F}_{2}$.

Let $\mathcal{S}$ be a maximal totally singular subspace of $\left(R(L)^{k}, q_{L}^{k}\right)$. Let $\mathfrak{L}(\mathcal{S})$ be the inverse image of $\mathcal{S}$ with respect to the canonical map

$$
\varphi_{L}^{k}:\left(L^{*}\right)^{k} \rightarrow R(L)^{k}, \quad\left(v_{i}\right) \mapsto\left(\varphi_{L}\left(v_{i}\right)\right),
$$

that is

$$
\mathfrak{L}(\mathcal{S})=\left(\varphi_{L}^{k}\right)^{-1}(\mathcal{S}) .
$$

Then $\mathfrak{L}(\mathcal{S})$ is a lattice, and its rank is $n k$ since it contains $L^{k}$, where $L^{k}$ is the orthogonal direct sum of $k$ copies of $L$.

Theorem 3.10. (cf. $\mathrm{Gr}]$ ) Let $L$ be an even lattice satisfying (3.4) and let $\mathcal{S}$ be a maximal totally singular subspace of $\left(R(L)^{k}, q_{L}^{k}\right)$.

(1) The lattice $\mathfrak{L}(\mathcal{S})$ of rank $n k$ is even and unimodular.

(2) Assume that $\mathcal{S}$ satisfies (2.2). If $L$ has no vectors of norm 2 then so does $\mathfrak{L}(\mathcal{S})$.

Proof. Since $\mathcal{S}$ is totally singular, $\mathfrak{L}(\mathcal{S})$ is even. By the maximality of $\mathcal{S}$, we have $\operatorname{dim} \mathcal{S}=$ $m k / 2$. Hence the determinant of $\mathfrak{L}_{L}(\mathcal{S})$ is $2^{m k / 2-m k / 2}=1$, which shows that $\mathfrak{L}(\mathcal{S})$ is unimodular.

By the definition of the map $w: R(L) \rightarrow\{0,1,2\}$, the minimum norm of $v+L \in R(L)$ is greater than or equal to $w(v+L)$. Hence for $u \in R(L)^{k}$ the minimum norm of $\left(\varphi_{L}^{k}\right)^{-1}(u)$ is greater than or equal to $w^{k}(u)$. Thus (2) follows from (2.2) and the assumption on $L$.

Remark 3.11. In general, the minimum norm of $\lambda+L \in R(L)$ may not be equal to $w(\lambda+L)$.

Remark 3.12. Let $L$ be an even lattice of rank $n \in 8 \mathbb{Z}$ satisfying (3.4). Let $J$ and $K$ be even unimodular lattices of rank $n$ satisfying $J \cap K \supset L$. Then $\Phi=\varphi_{L}(J)$ and $\Psi=\varphi_{L}(K)$ are maximal totally singular subspaces of $R(L)$. By (2.3) $\mathfrak{L}(\mathcal{S}(\Phi, \Psi ; k))$ is described in terms of $J$ and $K$ as follows:

$$
\mathfrak{L}(\mathcal{S}(\Phi, \Psi ; k))=\left\{\left(a_{1}+b, a_{2}+b, \ldots, a_{k}+b\right) \in \mathbb{R}^{n k} \mid a_{i} \in J, b \in K, \sum_{i=1}^{k} a_{i} \in J \cap K\right\}
$$

Note 3.13. In $[\mathrm{Gr}], \mathfrak{L}(\mathcal{S}(\Phi, \Psi ; k))$ was constructed and its minimum norm was studied. 


\subsection{Lepowsky and Meurman's description of the Leech lattice}

Let $E_{8}$ denote the $E_{8}$ root lattice. In this subsection, we consider the case where $k=3$ and $L=\sqrt{2} E_{8}$, and describe the Leech lattice. Moreover, as an application of Section 2.2, we study the Leech lattice and the Conway group. Note that the method in this subsection was already considered in [LM82, Gr99]. We continue the notation of the previous subsection.

Since $E_{8}$ is even, $L$ has no vectors of norm 2. By Lemma 3.9 (1), $L$ satisfies (3.4). Let $\mathcal{S}$ be a maximal totally singular subspace of $\left(R(L)^{3}, q_{L}^{3}\right)$ satisfying (2.2). Note that such a subspace exists by Proposition 2.4. By Theorem $3.10, \mathfrak{L}(\mathcal{S})$ is an even unimodular lattice of rank 24 without vectors of norm 2. It was shown in Co69 that such a lattice is isomorphic to the Leech lattice.

Corollary 3.14. Let $L=\sqrt{2} E_{8}$ and let $\mathcal{S}$ be a maximal totally singular subspace of $\left(R(L)^{3}, q_{L}^{3}\right)$ satisfying (2.2). Then $\mathfrak{L}(\mathcal{S})$ is isomorphic to the Leech lattice.

Remark 3.15. Let $\Phi$ and $\Psi$ be maximal totally singular subspaces of $\left(R(L), q_{L}\right)$ satisfying $\Phi \cap \Psi=0$. Then $\mathfrak{L}(\mathcal{S}(\Phi, \Psi ; 3))$ is isomorphic to the Leech lattice, which was shown in [LM82, Corollary 2.3]). Note that $\varphi_{L}^{-1}(\Phi)$ and $\varphi_{L}^{-1}(\Psi)$ are isomorphic to the $E_{8}$ root lattice.

Since $\operatorname{Aut}\left(L^{*}\right)=\operatorname{Aut}(L)$, there is a canonical homomorphism of groups

$$
\psi_{L}: \operatorname{Aut}\left(L^{*}\right) \rightarrow O\left(R(L), q_{L}\right) .
$$

Moreover, the center $Z(\operatorname{Aut}(L))$ of $\operatorname{Aut}(L)$ is generated by the -1-isometry, and the quotient group $\operatorname{Aut}(L) / Z(\operatorname{Aut}(L))$ is isomorphic to $O\left(R(L), q_{L}\right)$ (cf. [CS99]). Hence $\psi_{L}$ is surjective and $\operatorname{Ker} \psi_{L}=Z(\operatorname{Aut}(L))$.

Since the automorphism group of $L^{3}$ is isomorphic to $\operatorname{Aut}(L) 2 \operatorname{Sym}_{3}$ and $\operatorname{Aut}\left(L^{3}\right)=$ $\operatorname{Aut}\left(\left(L^{*}\right)^{3}\right), \psi_{L}$ induces a homomorphism of groups

$$
\psi_{L}^{3}: \operatorname{Aut}\left(\left(L^{*}\right)^{3}\right) \rightarrow \operatorname{Aut}\left(R(L)^{3}, w^{3}\right)
$$

By Proposition 2.2, $\psi_{L}^{3}$ is surjective. It follows from $\operatorname{Ker} \psi_{L}=Z(\operatorname{Aut}(L))$ that $\operatorname{Ker} \psi_{L}^{3}=$ $Z(\operatorname{Aut}(L))^{3} \cong 2^{3}$. Note that $\psi_{L}^{3}$ is compatible with $\varphi_{L}^{3}$, that is, $\varphi_{L}^{3}(g(v))=\psi_{L}^{3}(g)\left(\varphi_{L}^{3}(v)\right)$ for $g \in \operatorname{Aut}\left(\left(L^{*}\right)^{3}\right)$ and $v \in\left(L^{*}\right)^{3}$.

As corollaries of Proposition 2.5 and Theorem [2.8, we obtain the following well-known properties of $\operatorname{Aut}(\mathfrak{L}(\mathcal{S}))$.

Corollary 3.16. Gr99 Let $L=\sqrt{2} E_{8}$ and let $\mathcal{S}$ be a maximal totally singular subspace of $\left(R(L)^{3}, q_{L}^{3}\right)$ satisfying (2.2). Let $G$ be the stabilizer of $L^{3}$ in the automorphism group of $\mathfrak{L}(\mathcal{S})$.

(1) The automorphism group of $\mathfrak{L}(\mathcal{S})$ is transitive on the set of all sublattices of $\mathfrak{L}(\mathcal{S})$ isomorphic to the orthogonal direct sum of three copies of $\sqrt{2} E_{8}$.

(2) $G$ is isomorphic to an extension of the stabilizer of $\mathcal{S}$ in $\operatorname{Aut}\left(R(L)^{3}, w^{3}\right)$ by an elementary abelian 2-group of order $2^{3}$, and $G$ has the shape $2^{3} \cdot\left(2^{12}:\left(\mathrm{SL}_{4}(2) \times \mathrm{Sym}_{3}\right)\right)$.

Proof. Let $J$ be a sublattice of $\mathfrak{L}(\mathcal{S})$ isomorphic to $L^{3}$. It suffices to show that there is an automorphism of $\mathfrak{L}(\mathcal{S})$ which sends $J$ to $L^{3}$. Let $g$ be an orthogonal transformation of $\mathbb{R}^{24}$ such that $g(J)=L^{3}$. Then $g(\mathfrak{L}(\mathcal{S}))$ is an even unimodular lattice of rank 24 without vectors of norm 2 and contains $L^{3}$. Hence $\varphi_{L}^{3}(g(\mathfrak{L}(\mathcal{S})))$ is a maximal totally singular subspace of $R(L)^{3}$ satisfying (2.2). Then by Theorem 2.8, there exists $h \in \operatorname{Aut}\left(R(L)^{3}, w^{3}\right)$ such that $h\left(\psi_{L}^{3}(g(\mathfrak{L}(\mathcal{S})))\right)=\mathcal{S}$. Since $\varphi_{L}^{3}$ is surjective and compatible with $\psi_{L}^{3}$, there exists $\tilde{h} \in \operatorname{Aut}\left(L^{3}\right)$ such that $\tilde{h} \circ g(\mathfrak{L}(\mathcal{S}))=\mathfrak{L}(\mathcal{S})$. Since $\tilde{h} \circ g(J)=L^{3}$, we have proved (1). 
Clearly, $G$ is equal to the stabilizer of $\mathfrak{L}(\mathcal{S})$ in $\operatorname{Aut}\left(\left(L^{*}\right)^{3}\right)$. Since $\psi_{L}^{3}$ is compatible with $\varphi_{L}^{3}$ and $\varphi_{L}^{3}(\mathfrak{L}(\mathcal{S}))=\mathcal{S}, \psi_{L}^{3}(G)$ is the stabilizer of $\mathcal{S}$ in $\operatorname{Aut}\left(R(L)^{3}, w^{3}\right)$. Moreover, $\psi_{L}^{3}(G)$ is isomorphic to the stabilizer of $\mathcal{S}(\Phi, \Psi ; 3)$ in $\operatorname{Aut}\left(R(L)^{3}, w^{3}\right)$ by $(1)$. Since $\operatorname{Ker} \psi_{L}^{3} \cong 2^{3}$, we obtain (2) by Proposition 2.5.

Note 3.17. Corollary 3.16 (1) and (2) were shown in [Gr99, Proposition 3.5] and [Gr99, Corollary 3.10] respectively by a similar approach.

Let us count the number of vectors of norm 4 in $\mathfrak{L}(\mathcal{S})$. The following lemma is easy.

Lemma 3.18. Let $u \in R(L)$.

(1) If $w(u)=0$ then the number of vectors of norm 4 in $\varphi_{L}^{-1}(u)$ is 240 .

(2) If $w(u)=1$ then the number of vectors of norm 1 in $\varphi_{L}^{-1}(u)$ is 2 .

(3) If $w(u)=2$ then the number of vectors of norm 2 in $\varphi_{L}^{-1}(u)$ is 16 .

Proposition 3.19. Let $L=\sqrt{2} E_{8}$ and let $\mathcal{S}$ be a maximal totally singular subspace of $\left(R(L)^{3}, q_{L}^{3}\right)$ satisfying (2.2). Then the number of vectors of norm 4 in $\mathfrak{L}(\mathcal{S})$ is 196560.

Proof. By Theorem 2.8 and the surjectivity of $\psi_{L}^{3}$, we may assume that $\mathcal{S}=\mathcal{S}(\Phi, \Psi ; 3)$. Any vector of norm 4 in $\mathfrak{L}(\mathcal{S})$ belongs to $\left(\varphi_{L}^{3}\right)^{-1}(0)$ or $\left(\varphi_{L}^{3}\right)^{-1}(u)$ for some $u \in R(L)^{3}$ with $w^{3}(u)=4$. Note that $\left(\varphi_{L}^{3}\right)^{-1}(0)=L^{3}$. Hence by Lemmas 2.6 and 3.18, the number of vectors of norm 4 in $\mathfrak{L}(\mathcal{S})$ is

$$
(3 \times 240)+\left(3 \times\left(2^{4}-1\right)\right) \times 2^{0} \times 16^{2}+\left(3 \times\left(2^{4}-1\right) \times 2^{6}\right) \times 2^{2} \times 16^{1}=196560 .
$$

\section{Application to vertex operator algebras}

In this section, applying the results in Section 2 to VOAs, we obtain the moonshine VOA as a simple current extension of the tensor product of three copies of $V_{\sqrt{2} E_{8}}^{+}$and describe some automorphism group of the moonshine VOA.

The main approach in this section is to deduce the argument of VOAs to that of quadratic spaces in Section 2, which is quite similar to the approaches in Section 3 for binary codes and lattices. For example, the transitivity of the automorphism group of the moonshine VOA on the set of subVOAs isomorphic to $\left(V_{\sqrt{2} E_{8}}^{+}\right)^{3}$ will be shown in Theorem 4.17 by using the uniqueness of certain maximal totally singular subspaces in Theorem 2.8, which is similar to Corollaries 3.6 and 3.16 .

For the definitions and facts on VOAs and modules, see Section 1.

\subsection{A construction of holomorphic VOAs}

Let $V$ be a simple rational $C_{2}$-cofinite VOA of CFT type of central charge $n$. Let $R(V)$ denote the set of all isomorphism classes of irreducible $V$-modules. We consider the following conditions:

(a) Any irreducible $V$-module is a self-dual simple current module.

(b) For any $[M] \in R(V) \backslash\{[V]\}$, the lowest weight of $M$ belongs to $\mathbb{Z}_{>0} / 2$. 
(c) Let $q_{V}: R(V) \rightarrow \mathbb{F}_{2}$ be the map defined by setting $q_{V}([M])=0$ if $M$ is $\mathbb{Z}$-graded, and $q_{V}([M])=1$ if $M$ is $(\mathbb{Z}+1 / 2)$-graded. Then $\left(R(V), q_{V}\right)$ is a non-singular quadratic space of plus type over $\mathbb{F}_{2}$.

(d) For any irreducible $V$-module, the invariant bilinear form on it is symmetric.

Remark 4.1. The assumption (b) corresponds to (3.2) in binary codes and (3.4) in lattices.

If $V$ satisfies (a) then by Lemma $1.8 R(V)$ is an elementary abelian 2-group under the fusion rules. In this case, we view $R(V)$ as an $m$-dimensional vector space over $\mathbb{F}_{2}$, where $|R(V)|=2^{m}$.

Lemma 4.2. [DM97] (cf. [Sh07, Proposition 1.1]) Let $V$ be a simple rational $C_{2}$-cofinite VOA of CFT type satisfying (a). Let $X$ be a simple VOA containing $V$ as a full subVOA. Then the multiplicity of any irreducible $V$-submodule of $X$ is 1 , and $X$ is a simple current extension of $V$.

Lemma 4.3. [LY08, Theorem 1] Let $V$ be a simple rational $C_{2}$-cofinite VOA of CFT type satisfying (a). Let $X=\bigoplus_{[M] \in \mathcal{T}} M$ be a simple current extension of $V$, where $\mathcal{T}$ is a subgroup of $R(V)$. Let $U$ be an irreducible $X$-module and let $N$ be an irreducible $V$-submodule of $U$. Set

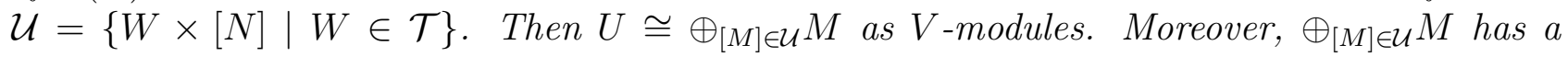
unique irreducible $X$-module structure extending its $V$-module structure up to isomorphism.

If $V$ satisfies (a)-(c) then the form $\langle\rangle:, R(V) \times R(V) \rightarrow \mathbb{F}_{2}$ defined by setting

$$
\left\langle W, W^{\prime}\right\rangle=q_{V}(W)+q_{V}\left(W^{\prime}\right)+q_{V}\left(W \times W^{\prime}\right)
$$

is non-singular and symplectic.

Let $V^{k}$ be the tensor product of $k$ copies of $V$. Then $V^{k}$ is a simple rational $C_{2}$-cofinite VOA of CFT type of central charge $n k$ ([ABD04, DMZ94, FHL93]). By Lemma 1.4, if $V$ satisfies (a)-(d) then so does $V^{k}$, and $\left(R(V)^{k}, q_{V}^{k}\right)=\left(R\left(V^{k}\right), q_{V^{k}}\right)$. Let $\mathcal{T}$ be a subset of $R(V)^{k}$ and let $\mathfrak{V}(\mathcal{T})$ denote the $V^{k}$-module defined by

$$
\mathfrak{V}(\mathcal{T})=\bigoplus_{[M] \in \mathcal{T}} M
$$

where we identify $([M(1)],[M(2)], \ldots,[M(k)]) \in R(V)^{k}$ with the isomorphism class of irreducible $V^{k}$-module $\otimes_{i=1}^{k} M(i)$ by Lemma 1.4 .

Proposition 4.4. Let $V$ be a simple rational $C_{2}$-cofinite VOA of CFT type satisfying $(a)-(d)$ and let $\mathcal{T}$ be a subset of $R(V)^{k}$. Then $\mathfrak{V}(\mathcal{T})$ has a simple VOA structure extending its $V^{k}$ module structure if and only if $\mathcal{T}$ is a totally singular subspace. Moreover, $\mathfrak{V}(\mathcal{T})$ is holomorphic if and only if $\mathcal{T}$ is a maximal totally singular subspace.

Proof. Recall that $V^{k}$ is a simple rational $C_{2}$-cofinite VOA of CFT type satisfying (a)-(d). By (c), $\mathfrak{V}(\mathcal{T})$ is $\mathbb{Z}$-graded if and only if $\mathcal{T}$ is totally singular. Hence the first assertion follows from Proposition 1.9 and $(\mathrm{d})$.

Assume that $\mathfrak{V}(\mathcal{T})$ is holomorphic. Let $\mathcal{U}$ be a totally singular subspace of $R(V)^{k}$ containing $\mathcal{T}$. Then by the first assertion and Proposition 1.5, the VOA $\mathfrak{V}(\mathcal{U})$ containing $\mathfrak{V}(\mathcal{T})$ as a full subVOA. Note that both $\mathfrak{V}(\mathcal{U})$ and $\mathfrak{V}(\mathcal{T})$ are of CFT type by (b). Since $\mathfrak{V}(\mathcal{T})$ is holomorphic, $\mathfrak{V}(\mathcal{U})=\mathfrak{V}(\mathcal{T})$, and hence $\mathcal{U}=\mathcal{T}$.

Assume that $\mathcal{T}$ is a maximal totally singular subspace. Let $U$ be an irreducible $\mathfrak{V}(\mathcal{T})$ -

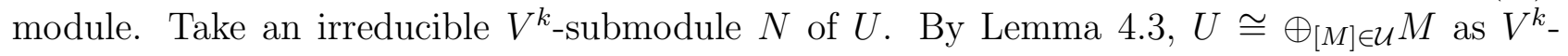
modules, where $\mathcal{U}=\{W \times[N] \mid W \in \mathcal{T}\}$. Let $W^{\prime} \in \mathcal{T}$. Then $W^{\prime} \times[N] \in \mathcal{U}$. Since $U$ is irreducible, $q_{V}^{k}([N])=q_{V}^{k}\left(W^{\prime} \times[N]\right)$. By $q_{V}^{k}\left(W^{\prime}\right)=0$ we have $\left\langle W^{\prime},[N]\right\rangle=q_{V}^{k}\left(W^{\prime}\right)+q_{V}^{k}([N])+$ $q_{V}^{k}\left(W^{\prime} \times[N]\right)=0$. Since $\mathcal{T}$ is maximal, we have $\mathcal{T}^{\perp}=\mathcal{T}$ by Lemma 1.1 $(1)$, and hence $[N] \in \mathcal{T}$. Thus $\mathcal{T}=\mathcal{U}$, and $U \cong \mathfrak{V}(\mathcal{T})$ as $\mathfrak{V}(\mathcal{T})$-modules by Lemma 4.3. 
Remark 4.5. By Proposition 1.5, the VOA structure on $\mathfrak{V}(\mathcal{T})$ extending its $V^{k}$-module structure is uniquely determined by $\mathcal{T}$ up to isomorphism.

Theorem 4.6. Let $V$ be a simple rational $C_{2}$-cofinite VOA of CFT type of central charge $n$ satisfying $(a)-(d)$. Let $\mathcal{S}$ be a maximal totally singular subspace of $\left(R(V)^{k}, q_{V}^{k}\right)$.

(1) $V^{k}$-module $\mathfrak{V}(\mathcal{S})$ has a holomorphic VOA structure of central charge nk extending its $V^{k}$-module structure.

(2) Assume that $\mathcal{S}$ satisfies (2.2). If $V_{1}=0$ then $\mathfrak{V}(\mathcal{S})_{1}=0$.

Proof. The first assertion follows from Proposition 4.4.

Let $[M] \in R(V)$. By the definition of the map $w: R(V) \rightarrow\{0,1,2\}$ and (b), the lowest weight of $M$ is greater than or equal to $w([M]) / 2$. Hence for $[N] \in R\left(V^{k}\right)$ the lowest weight of $N$ is greater than or equal to $w^{k}([N]) / 2$. Thus the latter assertion follows from (2.2) and $V_{1}=0$.

Remark 4.7. In general, the lowest weight of $M$ for $[M] \in R(V)$ may not be equal to $w([M]) / 2$.

Remark 4.8. The VOA $\mathfrak{V}(\mathcal{S})$ is rational by [Li97], $C_{2}$-cofinite by [ABD04] and of CFT by the assumption (b).

Remark 4.9. Let $V$ be a simple rational $C_{2}$-cofinite VOA of CFT type satisfying (a)-(d). Let $X$ and $N$ be holomorphic VOAs containing $V$ as a full subVOA. Then by Lemma 4.2 $X \cong \oplus_{[M] \in \Phi} M$ and $N \cong \oplus_{[M] \in \Psi} M$ as $V$-modules, where $\Phi$ and $\Psi$ are maximal totally singular subspaces of $R(V)$. By (2.3) $\mathfrak{V}(\mathcal{S}(\Phi, \Psi ; k))$ is described as follows:

$$
\mathfrak{V}(\mathcal{S}(\Phi, \Psi ; k))=\bigoplus_{\substack{[A(i)] \in \Phi,[B] \in \Psi \\ \Pi_{i=1}^{k}[A(i)] \in \Phi \cap \Psi}} \otimes_{i=1}^{k}(A(i) \times B) .
$$

\subsection{A description of the moonshine VOA by using $V_{\sqrt{2} E_{8}}^{+}$}

In this subsection, we consider the case where $k=3$ and $V=V_{\sqrt{2} E_{8}}^{+}$, and describe the moonshine VOA as a simple current extension of $V^{3}$. We continue the notation of the previous subsection. For the detail of $V$, see Section 1.4.

Let us describe the moonshine VOA $V^{\natural}$. Since $\sqrt{2} E_{8}$ has no vectors of norm 2 , we have $V_{1}=0$. By Lemma 1.10, $V$ is a simple rational $C_{2}$-cofinite VOA of CFT type of central charge 8 and satisfies (a)-(d) in the previous subsection. Let $\mathcal{S}$ be a maximal totally singular subspace of $\left(R(V)^{3}, q_{V}^{3}\right)$ satisfying (2.2). Note that such a subspace exists by Proposition 2.4. By Theorem 4.6, $\mathfrak{V}(\mathcal{S})$ is a holomorphic VOA of central charge 24 with $\mathfrak{V}(\mathcal{S})_{1}=0$. Since $V$ is framed, so is $\mathfrak{V}(\mathcal{S})$. It was shown in [LY07] that any holomorphic framed VOA without weight 1 subspace is isomorphic to the moonshine VOA $V^{\natural}$. Hence we obtain the following theorem.

Theorem 4.10. Let $V=V_{\sqrt{2} E_{8}}^{+}$and let $\mathcal{S}$ be a maximal totally singular subspace of $\left(R(V)^{3}, q_{V}^{3}\right)$ satisfying (2.2). Then the VOA $\mathfrak{V}(\mathcal{S})$ is isomorphic to the moonshine VOA $V^{\natural}$.

Remark 4.11. Let $X$ and $N$ be holomorphic VOAs of central charge 8 containing $V$ as a full subVOA. Then by Lemma 4.2 $X \cong \oplus_{[M] \in \Phi} M$ and $N \cong \oplus_{[M] \in \Psi} M$ as $V$-modules, where $\Phi$ and $\Psi$ are maximal totally singular subspaces of $R(V)$. Assume that $\Phi \cap \Psi=\{[V]\}$. Then by Proposition 2.4 and Theorem 4.10,

$$
V^{\natural} \cong \mathfrak{V}(\mathcal{S}(\Phi, \Psi ; 3))=\bigoplus_{\substack{[A(i)] \in \Phi,[B] \in \Psi \\[A(1)] \times[A(2)] \times[A(3)]=[V]}}(A(1) \times B) \otimes(A(2) \times B) \otimes(A(3) \times B) .
$$


as VOAs. Note that $X$ and $N$ are isomorphic to the lattice VOA $V_{E_{8}}$ associated to the $E_{8}$ root lattice by DM04b].

Remark 4.12. Since the Leech lattice $\Lambda$ contains $\left(\sqrt{2} E_{8}\right)^{3}$ as a sublattice, the VOA $V_{\Lambda}^{+}$contains $V^{3}$ as a full subVOA, and so does $V^{\natural}$ ([FLM88]). By the decomposition of $V^{\text {h }}$ into irreducible $V^{3}$-modules, one can directly find complementary maximal totally singular subspaces $\Phi_{0}$ and $\Psi_{0}$ of $R(V)$ such that $\mathfrak{V}\left(\mathcal{S}\left(\Phi_{0}, \Psi_{0} ; 3\right)\right) \cong V^{\natural}$ as $V^{3}$-modules. Hence Theorem 4.10 follows from Lemmas 1.1 (5), 1.6, 1.10 (4), Propositions 1.5, 4.16 (see next subsection) and Theorem 2.8. Note that this argument is independent of [LY07].

Note 4.13. In Mi04, the moonshine VOA was constructed as an extension of $V^{3}$.

As an application of our description, let us count the dimension of the weight 2 subspace of $\mathfrak{V}(\mathcal{S})$. The following lemma is easy (cf. [AD04]).

Lemma 4.14. Let $[M] \in R(V)$.

(1) If $w([M])=0$ then the dimension of the weight 2 subspace of $M$ is 156 .

(2) If $w([M])=1$ then the dimension of the weight $1 / 2$ subspace of $M$ is 1 .

(3) If $w([M])=2$ then the dimension of the weight 1 subspace of $M$ is 8 .

Proposition 4.15. Let $V=V_{\sqrt{2} E_{8}}^{+}$and let $\mathcal{S}$ be a maximal totally singular subspace of $\left(R(V)^{3}, q_{V}^{3}\right)$ satisfying (2.2). Then the dimension of the weight 2 subspace of $\mathfrak{V}(\mathcal{S})$ is 196884.

Proof. By Proposition 4.16 (see next subsection), $\operatorname{Aut}\left(V^{3}\right) \cong \operatorname{Aut}\left(R(V)^{3}, w^{3}\right)$. Hence by Lemma 1.6 and Theorem [2.8, we may assume that $\mathcal{S}=\mathcal{S}(\Phi, \Psi ; 3)$. The weight 2 subspace of $\mathfrak{V}(\mathcal{S})$ is the direct sum of those of $V^{3}$ and irreducible $V^{3}$-modules $M$ with $w^{3}([M])=4$. Hence by Lemmas 2.6 and 4.14, the dimension of the weight 2 subspace of $\mathfrak{V}(\mathcal{S})$ is

$$
(3 \times 156)+\left(3 \times\left(2^{5}-1\right)\right) \times 1^{0} \times 8^{2}+\left(3 \times\left(2^{5}-1\right) \times 2^{8}\right) \times 1^{2} \times 8^{1}=196884 .
$$

\subsection{An $E_{8}$-approach to the automorphism group of $V^{\natural}$}

In this subsection, as an application of Sections 2.2 and 4.2, we study the automorphism group of the moonshine VOA $V^{\natural}$. Note that the results in this section are independent of the fact that the automorphism group of $V^{\natural}$ is isomorphic to the Monster. We continue the notation of the previous subsection.

First, we determine the automorphism group of $V^{k}$.

Proposition 4.16. Let $V=V_{\sqrt{2} E_{8}}^{+}$. Then the automorphism group of $V^{k}$ is isomorphic to $\operatorname{Aut}(V) \gtrless \operatorname{Sym}_{k}$.

Proof. Clearly, Aut $(V) \imath \operatorname{Sym}_{k}$ acts on $V^{k}$ as an automorphism group.

Let $g \in \operatorname{Aut}\left(V^{k}\right)$. By Lemma 1.4, $R\left(V^{k}\right)$ is identified with $R(V)^{k}$. By Lemma 1.10 (3), $w^{k}([M]) / 2$ is the lowest weight of $M$ for any irreducible $V^{k}$-module $M$. Hence by Lemma $1.2(2) g \in \operatorname{Aut}\left(R(V)^{k}, w^{k}\right)$. By Lemma 1.10 (4) and Proposition 2.2, $\operatorname{Aut}\left(R(V)^{k}, w^{k}\right) \cong$ $O\left(R(V), q_{V}\right) \prec \operatorname{Sym}_{k} \cong \operatorname{Aut}(V) \prec \operatorname{Sym}_{k}$. Hence $g \in \operatorname{Aut}(V) \prec \operatorname{Sym}_{k}$. 
Next, we consider lifts of symmetries of the quadratic space. Let $G$ be the automorphism group of $\mathfrak{V}(\mathcal{S})$. Recall that $\mathfrak{V}(\mathcal{S})$ is a simple current extension of $V^{3}$ graded by $\mathcal{S}$. By (1.1), we obtain a homomorphism of groups

$$
\eta: N_{G}\left(\mathcal{S}^{*}\right) \rightarrow\left\{g \in \operatorname{Aut}\left(V^{3}\right) \mid g \circ \mathcal{S}=\mathcal{S}\right\}
$$

By Proposition 1.7, $\eta$ is surjective and Ker $\eta=\mathcal{S}^{*} \cong 2^{15}$. We now prove the following theorem without properties of the Monster.

Theorem 4.17. Let $V=V_{\sqrt{2} E_{8}}^{+}$and let $\mathcal{S}$ be a maximal totally singular subspace of $\left(R(V)^{3}, q_{V}^{3}\right)$ satisfying (2.2). Let $G$ be the automorphism group of $\mathfrak{V}(\mathcal{S})$ and let $H$ be the stabilizer of $V^{3}$ in $G$.

(1) $H=N_{G}\left(\mathcal{S}^{*}\right)$.

(2) $G$ is transitive on the set of all full subVOAs of $\mathfrak{V}(\mathcal{S})$ isomorphic to the tensor product of three copies of $V_{\sqrt{2} E_{8}}^{+}$.

(3) $H$ is isomorphic to an extension of the stabilizer of $\mathcal{S}$ in $\operatorname{Aut}\left(R(V)^{3}, w^{3}\right)$ by an elementary abelian 2-group of order $2^{15}$, and $H$ has the shape $2^{15} \cdot\left(2^{20}:\left(\mathrm{SL}_{5}(2) \times \operatorname{Sym}_{3}\right)\right)$.

Proof. Since the subspace of $\mathfrak{V}(\mathcal{S})$ fixed by $\mathcal{S}^{*}$ is $V^{3}$, the normalizer $N_{G}\left(\mathcal{S}^{*}\right)$ is a subgroup of $H$. Since $\mathfrak{V}(\mathcal{S})$ is a simple current extension of $V^{3}$, the multiplicity of any irreducible $V^{3}$ submodule is 1 . Hence $H$ acts on the set of irreducible $V^{3}$-submodules as permutations, and $H$ is a subgroup of the normalizer, which proves (1).

Let $X$ be a full subVOA of $\mathfrak{V}(\mathcal{S})$ isomorphic to $V^{3}$. It suffices to show that there is an automorphism of $\mathfrak{V}(\mathcal{S})$ which sends $X$ to $V^{3}$. By Lemma 4.2, $\mathfrak{V}(\mathcal{S})$ is a simple current extension of $X$. Let $\mathcal{T}$ be the set of the isomorphism classes of irreducible $X$-submodules of $\mathfrak{V}(\mathcal{S})$. Then by Proposition 4.4, $\mathcal{T}$ is a maximal totally singular subspace of $R(X)\left(\cong R\left(V^{3}\right)\right)$. Since the weight 1 subspace of $\mathfrak{V}(\mathcal{S})$ is trivial, $\mathcal{T}$ satisfies $(2.2)$. Hence by Theorem 2.8 there exists $g \in \operatorname{Aut}(X)\left(\cong \operatorname{Aut}\left(R(V)^{3}, w^{3}\right)\right)$ such that $g \circ \mathcal{T}=\mathcal{S}$. By Lemma 1.6, $g \circ \mathfrak{V}(\mathcal{S})$ has a VOA structure isomorphic to $\mathfrak{V}(\mathcal{S})$ and let $\tilde{g}$ be the isomorphism of $\operatorname{VOAs}$ from $\mathfrak{V}(\mathcal{S})$ to $g \circ \mathfrak{V}(\mathcal{S})$. Note that the set of the isomorphism classes of irreducible submodule of $g \circ \mathfrak{V}(\mathcal{S})$ for $\tilde{g}(X) \cong V^{3}$ is $g \circ \mathcal{T}=\mathcal{S}$. Hence by Proposition 1.5, there is an isomorphism of VOAs $h$ from $g \circ \mathfrak{V}(\mathcal{S})$ to $\mathfrak{V}(\mathcal{S})$ such that $h \circ \tilde{g}(X)=V^{3}$. Thus we obtain an automorphism $h \circ \tilde{g}$ of $\mathfrak{V}(\mathcal{S})$ such that $h \circ \tilde{g}(X)=V^{3}$, which proves $(2)$.

By Proposition 4.16, Aut $\left(R(V)^{3}, w^{3}\right)=\operatorname{Aut}\left(V^{3}\right) \cong \operatorname{Aut}(V) \gtrless \operatorname{Sym}_{3}$. Hence (3) follows from (1), (2), Proposition 2.5 and (4.1).

Combining Theorems 4.10 and 4.17, we obtain the following corollary.

Corollary 4.18. Let $V^{\natural}$ be the moonshine VOA.

(1) The automorphism group of $V^{\natural}$ is transitive on the set of all full subVOAs isomorphic to $\left(V_{\sqrt{2} E_{8}}^{+}\right)^{3}$.

(2) The stabilizer of a full subVOA of $V^{\natural}$ isomorphic to $\left(V_{\sqrt{2} E_{8}}^{+}\right)^{3}$ in the automorphism group of $V^{\text {b }}$ has the shape $2^{15} \cdot\left(2^{20}:\left(\mathrm{SL}_{5}(2) \times \mathrm{Sym}_{3}\right)\right)$.

Remark 4.19. By the order of the Monster ([CCNPW85]) and the corollary above, the number of full subVOAs of $V^{\text {b }}$ isomorphic to $\left(V_{\sqrt{2} E_{8}}^{+}\right)^{3}$ is

$3^{17} \cdot 5^{8} \cdot 7^{5} \cdot 11^{2} \cdot 13^{3} \cdot 17 \cdot 19 \cdot 23 \cdot 29 \cdot 41 \cdot 47 \cdot 59 \cdot 71=391965121389536908413379198941796875$. 
Remark 4.20. Assume that $\mathcal{S}=\mathcal{S}(\Phi, \Psi ; 3)$. By Proposition 2.5, $N_{G}\left(\mathcal{S}^{*}\right)$ normalizes the elementary abelian 2-subgroup $\Psi_{(123)}^{*}$ of $\mathcal{S}^{*}$ of order $2^{5}$.

Remark 4.21. In [Sh07], $V^{\natural}$ was studied as a simple current extension of $V_{\Lambda(5)}^{+}$for a certain sublattice $\Lambda(5)$ of the Leech lattice containing $\left(\sqrt{2} E_{8}\right)^{3}$. One can find complementary maximal totally singular subspaces $\tilde{\Phi}$ and $\tilde{\Psi}$ of $R(V)$ satisfying

$$
V_{\Lambda(5)}^{+} \cong \oplus_{[M] \in \operatorname{Span}_{\mathbb{F}_{2}}\left\{\tilde{\Phi}_{(12)}, \tilde{\Phi}_{(13)}\right\}} M \quad \text { and } \quad \mathfrak{V}(\mathcal{S}(\tilde{\Phi}, \tilde{\Psi} ; 3)) \cong V^{\natural}
$$

as $V^{3}$-modules. Hence the normalizer of $\tilde{\Psi}_{(123)}^{*}\left(\cong 2^{5}\right)$ of shape $2^{5} \cdot 2^{16} \cdot 2^{2+12} \cdot \mathrm{Sym}_{3} . \mathrm{SL}_{5}(2)$ described in Sh07 is conjugate to $N_{G}\left(\mathcal{S}^{*}\right)$.

Note 4.22. In [Mi04, Lemma 9.3] a subgroup of shape $2^{7} \cdot 2^{20} \cdot 2^{12} \cdot\left(\mathrm{SL}_{4}(2) \times \mathrm{Sym}_{3}\right)$ of the stabilizer of $V^{3}$ in $\operatorname{Aut}\left(V^{\natural}\right)$ was described as the stabilizer of certain Virasoro frame of $V$ (cf. [LS09, Section 6.5]). By the shapes, it is a maximal subgroup of $N_{G}\left(\mathcal{S}^{*}\right)$.

Note 4.23. According to [CCNPW85, the subgroup of the Monster obtained in Theorem 4.17 is a maximal 2-local subgroup of shape $2^{5+10+20} \cdot\left(\mathrm{SL}_{5}(2) \times \mathrm{Sym}_{3}\right)$.

Acknowledgements. The author thanks to Professor Ching Hung Lam for helpful suggestions on this study. He also thanks to Professor Hiroshi Yamauchi for useful discussion on simple current extensions of VOAs. Part of the work was done when he was visiting Academia Sinica in 2009 and Imperial College London in 2010. He thanks the staff for their help.

\section{References}

[ABD04] T. Abe, G. Buhl and C. Dong, Rationality, regularity, and $C_{2}$-cofiniteness, Trans. Amer. Math. Soc. 356 (2004), 3391-3402.

[AD04] T. Abe and C. Dong, Classification of irreducible modules for the vertex operator algebra $V_{L}^{+}$: general case. J. Algebra 273 (2004), 657-685.

[ADL05] T. Abe, C. Dong, and H. Li, Fusion rules for the vertex operator algebra $M(1)$ and $V_{L}^{+}$, Comm. Math. Phys. 253 (2005), 171-219.

[Bo86] R.E. Borcherds, Vertex algebras, Kac-Moody algebras, and the Monster, Proc. Nat'l. Acad. Sci. U.S.A. 83 (1986), 3068-3071.

[Ch97] C.C. Chevalley, The algebraic theory of spinors and Clifford algebras, Springer-Verlag, Berlin, 1997

[Co69] J.H. Conway, A characterisation of Leech's lattice, Invent. Math. 7 (1969) 137-142.

[CCNPW85] J.H. Conway, R.T. Curtis, S.P. Norton, R.A. Parker and R.A. Wilson, Atlas of finite groups, Oxford, Oxford University Press 1985.

[CS99] J.H. Conway and N.J.A. Sloane, Sphere packings, lattices and groups, 3rd Edition, Springer, New York, 1999.

[DGH98] C. Dong, R.L. Griess, and G. Höhn, Framed vertex operator algebras, codes and Moonshine module, Comm. Math. Phys. 193 (1998), 407-448.

[DLM96] C. Dong, H. Li and G. Mason, Simple currents and extensions of vertex operator algebras, Comm. Math. Phys. 180 (1996), 671-707. 
[DM97] C. Dong and G. Mason, On quantum Galois theory, Duke Math. J. 86 (1997), 305-321.

[DM04] C. Dong and G. Mason, Rational vertex operator algebras and the effective central charge, Int. Math. Res. Not. (2004), 2989-3008.

[DM04b] C. Dong and G. Mason, Holomorphic vertex operator algebras of small central charge, Pacific J. Math. 213 (2004), 253-266.

[DMZ94] C. Dong, G. Mason and Y. Zhu, Discrete series of the Virasoro algebra and the moonshine module, Proc. Sympos. Pure Math. 56 (1994), 295-316.

[FHL93] I. Frenkel, Y. Huang and J. Lepowsky, On axiomatic approaches to vertex operator algebras and modules, Mem. Amer. Math. Soc. 1041993.

[FLM88] I. Frenkel, J. Lepowsky and A. Meurman, Vertex operator algebras and the Monster, Pure and Appl. Math., Vol.134, Academic Press, Boston, 1988.

[Gr98] R.L. Griess, A vertex operator algebra related to $E_{8}$ with automorphism group $\mathrm{O}^{+}(10,2)$, Ohio State Univ. Math. Res. Inst. Publ. 7 (1998), 43-58.

[Gr99] R.L. Griess, Pieces of eight: semiselfdual lattices and a new foundation for the theory of Conway and Mathieu groups. Adv. Math. 148 (1999), 75-104.

[Gr] R.L. Griess, Rank 72 high minimum norm lattices, preprint, arXiv:0910.2055, to appear in J. Number Theory.

[Hu96] Y. Huang, A nonmeromorphic extension of the moonshine module vertex operator algebra, Contemp. Math. (1996), 123-148.

[Hu05] Y. Huang, Differential equations and intertwining operators, Commun. Contemp. Math. 7 (2005), 375-400.

[Hö95] G. Höhn, Selbstduale Vertexoperatorsuperalgebren und das Babymonster, Dissertation, Rheinische Friedrich-Wilhelms-Universitat Bonn, Bonn, 1995.

[Hö08] G. Höhn, Conformal designs based on vertex operator algebras, Adv. Math. 217 (2008), 2301-2335.

[LS09] C. Lam and H. Shimakura, Frame stabilizers for framed vertex operator algebras associated to lattices having 4-frames, Int. Math. Res. Not. IMRN, 2009 (2009), 4547-4577

[LY07] C. Lam and H. Yamauchi, A characterization of the moonshine vertex operator algebra by means of Virasoro frames, Int. Math. Res. Not. IMRN (2007), ID rnm003, 10 pp.

[LY08] C. Lam and H. Yamauchi, On the structure of framed vertex operator algebras and their pointwise frame stabilizers, Comm. Math. Phys. 277 (2008), 237-285.

[LM82] J. Lepowsky and A. Meurman, An $E_{8}$-approach to the Leech lattice and the Conway group, J. Algebra 77 (1982), 484-504.

[Li97] H. Li, Extension of vertex operator algebras by a self-dual simple module, J. Algebra 187 (1997), 236-267.

[MS77] F.J. MacWilliams, and N.J.A. Sloane, The theory of error-correcting codes, North-Holland Mathematical Library, Vol. 16. North-Holland Publishing Co., Amsterdam-New YorkOxford, 1977

[Mi04] M. Miyamoto, A new construction of the Moonshine vertex operator algebra over the real number field, Ann. of Math. 159 (2004), 535-596. 
[SY03] S. Sakuma and H. Yamauchi, Vertex operator algebra with two Miyamoto involutions generating $S_{3}$, J. Algebra 267 (2003), 272-297.

[Sh04] H. Shimakura, The automorphism group of the vertex operator algebra $V_{L}^{+}$for an even lattice $L$ without roots, J. Algebra 280 (2004), 29-57.

[Sh07] H. Shimakura, Lifts of automorphisms of vertex operator algebras in simple current extensions, Math. Z. 256 (2007), 491-508.

[Sh] H. Shimakura, On isomorphism problems of VOAs associated with even lattices, preprint.

[Ve79] B.B. Venkov, Odd unimodular lattices, Algebraic numbers and finite groups, Zap. Naučn. Sem. Leningrad. Otdel. Mat. Inst. Steklov, 86 (1979), 40-48. 